\title{
Scaling law and reduced models for epitaxially strained crystalline films
}

\author{
M. Goldman * $\quad$ B. Zwicknagl ${ }^{\dagger}$
}

\begin{abstract}
A variational model for the epitaxial deposition of a film on a rigid substrate in the presence of a crystallographic misfit is studied. The scaling behavior of the minimal energy in terms of the volume of the film and the amplitude of the misfit is considered, and reduced models in the various regimes are derived by $\Gamma$-convergence methods. Depending on the relation between the thickness of the film and the amplitude of the misfit, the surface or the elastic energy contribution dominates, and in the critical case the two contributions balance. In particular, the formation of islands is proven if the amplitude of the misfit is large compared to the volume of the film.
\end{abstract}

\section{Introduction}

We study a free energy functional introduced in [42] (see also $[9,20,26]$ ) to model the epitaxial deposition of a film on a rigid substrate when there is a crystallographic misfit between the two solids. The energy consists of the stored elastic energy in the film and the interfacial energy of its free surface. Precisely, we consider

$$
F_{d, e_{0}}(u, h):=\int_{\Omega_{h}} W(\nabla u) d x d y+\int_{0}^{1} \sqrt{1+\left|h^{\prime}\right|^{2}} d x
$$

where the Lipschitz function $h:[0,1] \rightarrow[0, \infty)$ describes the profile of the deposited film, $\Omega_{h}:=\{(x, y): 0 \leq x \leq 1,0 \leq y \leq h(x)\}$, and $u \in W^{1, p}\left(\Omega_{h} ; \mathbb{R}^{2}\right)$ is the planar displacement. Note that we restrict ourselves to two-dimensional morphologies which correspond to three-dimensional configurations with planar symmetry (see Section 5 for some results in the higher-dimensional setting). We consider film profiles of fixed volume $d>0$, i.e., $\int_{0}^{1} h(x) d x=d$, and impose the boundary conditions $h(0)=h(1)=0$ (see Figure 1). The parameter $e_{0} \in \mathbb{R}$ stands for the amplitude of the crystallographic misfit between the film and the rigid substrate, and is introduced in this model via the boundary condition $u(x, 0)=e_{0}(x, 0)$. This condition forces the film to be strained. The free energy density $W$ is nonnegative and satisfies a $p$-growth condition (see Section 2.3). We note that the

\footnotetext{
*Max Planck Institute for Mathematics in the Sciences, Leipzig (Germany), email: goldman@mis.mpg.de

${ }^{\dagger}$ Institute for Applied Mathematics, Bonn University, Bonn (Germany), email: zwicknagl@iam.uni-bonn.de
} 


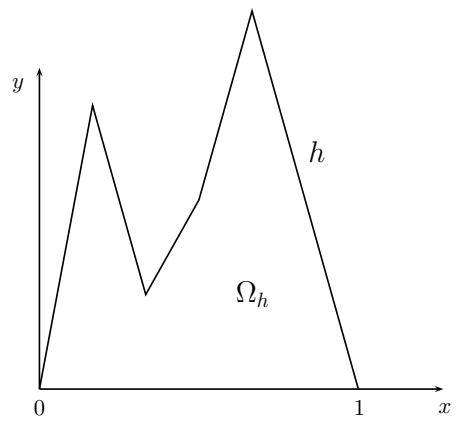

Figure 1: A height profile $h$

functional (1) is normalized by setting a typical surface energy per unit length to one, i.e., the material parameters are all collected in $W$.

It has been observed experimentally and numerically that the qualitative shape of the optimal profile depends on the volume $d$ of the film and on the amplitude of the misfit $e_{0}$ (see $[9,27,31,43])$. If the volume is small then the film typically forms a flat layer. If the volume of the film is sufficiently large then the flat configuration is no longer stable and atoms often tend to rearrange in such a way that part of the bulk energy is released. The competition between elastic and surface energy then gives rise to the formation of islands on the substrate surface. Such island formation is used for many optical and optoelectronic applications, such as quantum dot lasers.

In recent years, the mathematical analysis of the model (1) has been devoted to the geometrically linear small strain approximation (see [20, 26, 9]), corresponding to small deformations, in which $W$ depends only on the symmetrized gradient $E(u):=\frac{\nabla u+\nabla^{T} u}{2}$. Since we are mainly interested in the regime of large mismatch $e_{0}$, the small strain hypothesis is questionable and instead we focus on the geometrically nonlinear case. We point out that our analysis can be easily carried over to the geometrically linear setting with only very minor modifications. However, in the limit of large strains or large volumes, the model becomes physically less justified since dislocations, fracture, and other plastic effects are more likely to appear. Also, the hypothesis that the film remains a subgraph seems to be rather restrictive. Our aim is thus not to give a quantitative prediction of the formation of islands, but to show how the latter is encoded in this classical model and to highlight the underlying scales. We try in particular to better understand how the relation between the misfit and the volume of the deposited film drives the pattern formation.

Our main result is the following scaling law for the energy (see Theorem 3.1): There are positive constants $C_{1}$ and $C_{2}$ such that for all choices of parameters $d$ and $e_{0}$,

$$
C_{1} \max \left\{1, d, e_{0}^{p / 3} d^{2 / 3}\right\} \leq \inf F_{d, e_{0}} \leq C_{2} \max \left\{1, d, e_{0}^{p / 3} d^{2 / 3}\right\} .
$$

Subsequently, we consider the scaling regimes separately and derive reduced limiting func- 
tionals in the spirit of $\Gamma$-convergence in the three scaling regimes and for the transition regime $d \simeq e_{0}^{p}$. Our results agree qualitatively with the experimentally observed morphologies. Consider first large volumes $d \rightarrow+\infty$. If the amplitudes of the misfits $e_{0} \rightarrow+\infty$ are such that $\frac{e_{0}^{p}}{d} \rightarrow 0$, i.e., $\inf F_{d, e_{0}} \simeq d$, then the surface energy dominates, and the main energy contribution comes from the non-horizontal parts of the boundary. Indeed, in this case the limiting reduced functional does not depend on the elastic strain and charges only the vertical parts of the boundary of $\Omega_{h}$ (see Proposition 4.2). The limit problem is minimized by the flat configuration (see Lemma 2.6). In the transition regime in which $d \simeq e_{0}^{p} \rightarrow+\infty$, surface and elastic contributions compete (see Section 4.3). Consequently, the minimizer of the reduced model is either the flat configuration or an island, depending on the elastic properties of the material. Finally, if the amplitudes of the misfits are large compared to the volumes, i.e., if $e_{0} \rightarrow+\infty$ and $\frac{e_{0}^{p}}{d} \rightarrow+\infty$, then $\inf F_{d, e_{0}} \simeq e_{0}^{p / 3} d^{2 / 3}$, and the main contribution to the energy comes from the elastic part. Precisely, the limiting functional is now defined on the space of (at most) countable sums of Dirac masses. Using a compactness argument (see Proposition 4.7), this proves that in this regime, low-energy sequences have to converge to sums of Dirac masses. This shows that the formation of islands is energetically favorable in this regime. To the best of our knowledge, this work is the first rigorous mathematical justification of the formation of islands in relation to the amplitude of the mismatch between crystallographic lattices in epitaxially strained films.

For small volumes $d \rightarrow 0$ and small amplitudes of the misfits $e_{0} \rightarrow 0$ such that $e_{0}^{p} d^{2} \rightarrow 0$, we have inf $F_{d, e_{0}} \simeq 1$, and the limiting reduced functional is simply a constant (see Proposition 4.1). In particular, for low-energy sequences in this regime neither the elastic energy nor the non-horizontal parts of the boundary contribute significantly to the energy. If, however, $e_{0}^{p} d^{2} \rightarrow+\infty$, then $\inf F_{d, e_{0}} \simeq e_{0}^{p / 3} d^{2 / 3}$, and, as discussed above for the case of large volumes, low-energy sequences converge to sums of Dirac masses, and the formation of islands is energetically favorable. Notice that our proof makes fundamental use of the fact that we are on a bounded domain. However, building on the techniques developed here, we investigate with P. Bella the same type of problems on unbounded domains and obtain similar results (see [6]).

In the last part of the paper we adapt our method to prove the scaling law in higher dimension for the Dirichlet elastic energy (see Proposition 5.1). Even though our method seems to be quite flexible, the richness of the geometry makes the extension to more general energy functionals difficult (see also [13] for some results). We finally note that in higher dimension, the elastic part of the energy can be seen as a repulsive nonlocal term which relates the problem to the Ohta-Kawasaki model that has recently attracted a great deal of attention (see $[34,16,14,30,2])$.

We note that in the regimes in which the elastic energy is not dominated by the surface energy, the recession function of $W$ at infinity plays an essential role in the limit. The latter is defined by

$$
W^{\infty}(A):=\limsup _{t \rightarrow+\infty} \frac{W(t A)}{t^{p}} \text { for } A \in \mathbb{R}^{2 \times 2},
$$


and appears naturally in problems involving functionals with linear growth (see $[22,23])$.

Previous work, starting with [12], has been devoted to regularity and other qualitative properties of islands, once they are formed. In [20] the authors prove that minimizing configurations are smooth outside of a finite number of cusps and cuts. Subsequently, it is proven in [26] that for small values of the amplitude of the mismatch, the flat configuration is always minimizing no matter how thick is the film. Further, they proved that for larger (but fixed) values of $e_{0}$, there are increasing thresholds for the volume $d$ such that the flat configuration changes from being an absolute minimizer to being only a local minimizer, and such that it is no longer a local minimizer above a certain threshold for $d$. Finally, they showed that even though the flat configuration is no longer minimizing, there exists another threshold below which minimizers are smooth.

Our analysis bears similarities with dimension reduction problems (see [5, 19, 24, 25, 32, 37]). In contrast to these works, we study the behavior for small and for large volumes. Furthermore, the reference configuration is not given but is one of the unknowns. Another feature of (1) is the absence of regularizing second order terms which are often used to obtain scaling laws in elasticity (see $[35,17,7]$ ). Notice also that our proof of the lower bound for the scaling law in dimension two does not rely on interpolation inequalities.

Here, we deal only with the stationary setting, but we refer to $[21,40]$ for recent results on the time evolution problem. Finally, as pointed out above, it would be also interesting to investigate the model without the hypothesis that the domain of the film is a subgraph (see [11]). We expect to observe a qualitatively similar behavior where typical shapes of islands are not rectangles but balls.

The plan of the paper is as follows: In Section 2 we set the notation and collect some preliminary results concerning both the surface energy and the elastic energy. In Section 3 we establish the scaling law for $F_{d, e_{0}}$, and in Section 4 we derive and discuss reduced models in the various scaling regimes. Finally in Section 5, we present a partial extension of our results to higher dimensions.

\section{Notation and preliminary results}

Set $I:=[0,1]$, and throughout the text denote by $C$ and $c$ constants that may vary from expression to expression. For $\Omega \subset \mathbb{R}^{2}$, we denote by $\mathcal{H}^{1}(\Omega)$ its one-dimensional Hausdorff measure, and by $|\Omega|$ its two-dimensional Lebesgue measure. Given two sets $A, B \subset \mathbb{R}^{2}$, we define their Hausdorff distance as $d_{\mathcal{H}}(A, B):=\inf \{r>0: A \subset N(B, r)$ and $B \subset N(A, r)\}$, where $N(A, r):=\left\{x \in \mathbb{R}^{2}: d(x, A)<r\right\}$, and $d(x, A)$ denotes the distance from $x$ to $A$. For $c \in \mathbb{R}$, let $\delta_{c}$ be the Dirac measure at the point $c$. Given a vector $(x, y) \in \mathbb{R}^{2}$, we denote by $|(x, y)|:=\left(x^{2}+y^{2}\right)^{1 / 2}$ its Euclidean norm. For a matrix $A \in \mathbb{R}^{2 \times 2}$, we consider the Frobenius norm given by $|A|^{2}:=\operatorname{Tr}\left(A^{T} A\right)$. 


\section{$2.1 \quad \Gamma$-convergence}

We briefly recall the definition and some of the main properties of $\Gamma$-convergence (see $[18,10]$ ).

Definition 2.1. Let $X$ be a topological space. We say that a sequence of functions $\left\{F_{n}\right\}$, $F_{n}: X \rightarrow \mathbb{R}$, (sequentially) $\Gamma$-converges to $F: X \rightarrow \mathbb{R}$ and write $F_{n} \stackrel{\Gamma}{\rightarrow} F$ if the following two conditions are satisfied.

(i) If $\left\{x_{n}\right\} \subset X$ satisfies $\sup F_{n}\left(x_{n}\right)<+\infty$ then there exists a subsequence (not relabeled) such that $x_{n} \rightarrow x \in X$ and

$$
\liminf _{n \rightarrow+\infty} F_{n}\left(x_{n}\right) \geq F(x) .
$$

(ii) For every $x \in X$ there exists a sequence $\left\{x_{n}\right\} \subset X$ such that $x_{n} \rightarrow x$ and

$$
\limsup _{n \rightarrow+\infty} F_{n}\left(x_{n}\right) \leq F(x) .
$$

The main property of $\Gamma$-convergence is the following:

Theorem 2.2. Suppose that a sequence $\left\{F_{n}\right\}$ with $F_{n}: X \rightarrow \mathbb{R} \Gamma$-converges to $F$ and that there is some $C>0$ such that $\inf _{x \in X} F_{n}(x) \leq C$ for all $n \in \mathbb{N}$. Then

$$
\inf _{x \in X} F_{n}(x) \rightarrow \inf _{x \in X} F(x) .
$$

Moreover, if $x_{n}$ is a minimizer of $F_{n}$ then there is a subsequence of $\left\{x_{n}\right\}$ that converges to a minimizer of $F$.

\subsection{The Surface Energy}

We review some results from $[20,9]$ adapted to our setting. If $h: I \rightarrow[0,+\infty)$ is lower semicontinuous, then we set

$$
\Omega_{h}:=\{(x, y): 0 \leq x \leq 1,0 \leq y<h(x)\},
$$

and denote the pointwise variation of $h$ by

$$
\operatorname{Var} h:=\sup \left\{\sum_{i=1}^{n}\left|h\left(x_{i}\right)-h\left(x_{i-1}\right)\right|:\left\{x_{1}, \ldots, x_{n}\right\} \text { is a partition of } I\right\} .
$$

If Var $h$ is finite then $h$ is said to be of bounded pointwise variation (see [4]). If $h$ is a function of bounded pointwise variation then we define

$$
\begin{aligned}
& h^{-}(x):=\min \left\{h\left(x^{+}\right), h\left(x^{-}\right)\right\}=\liminf _{z \rightarrow x} h(z), \\
& h^{+}(x):=\max \left\{h\left(x^{+}\right), h\left(x^{-}\right)\right\}=\limsup _{z \rightarrow x} h(z),
\end{aligned}
$$


where $h\left(x^{ \pm}\right):=\lim _{z \rightarrow x^{ \pm}} h(z)$. Further, let

$$
\int_{0}^{1}\left|h^{\prime}\right|:=\operatorname{Var} h+h\left(0^{+}\right)+h\left(1^{-}\right) .
$$

We denote by $\Gamma_{\text {cuts }}$ the at most countable collection of vertical cuts,

$$
\Gamma_{\text {cuts }}:=\left\{(x, y): x \in(0,1) \cap S(h), h(x) \leq y \leq h^{-}(x)\right\},
$$

where $S(h):=\left\{x \in(0,1): h(x)<h^{-}(x)\right\}$. The following proposition is a slight adaption of results from [20, Proposition 2.2, Lemma 2.5, and Theorem 2.8].

Proposition 2.3. Let $\left\{h_{n}\right\}$ be a sequence of nonnegative Lipschitz functions such that

$$
\sup _{n} \int_{0}^{1} \sqrt{1+\left|h_{n}^{\prime}\right|^{2}} d x+\int_{0}^{1} h_{n} d x<+\infty \text {. }
$$

Then there exists a subsequence (not relabeled) such that

(i) $\mathbb{R}^{2} \backslash \Omega_{h_{n}}$ converges in the Hausdorff metric to $\mathbb{R}^{2} \backslash \Omega_{h}$ where

$$
h(x):=\inf \left\{\liminf _{n \rightarrow+\infty} h_{n}\left(x_{n}\right): x_{n} \rightarrow x\right\}
$$

is lower semicontinuous and of bounded pointwise variation;

(ii) $h_{n} \rightarrow h$ in $L^{1}(I)$;

(iii) for every sequence $\left\{a_{n}\right\} \subset \mathbb{R}^{+}$with $a_{n} \rightarrow 0$

$$
\liminf _{n \rightarrow+\infty} \int_{0}^{1} \sqrt{a_{n}+\left|h_{n}^{\prime}\right|^{2}} d x \geq \int_{0}^{1}\left|h^{\prime}\right|+2 \mathcal{H}^{1}\left(\Gamma_{\text {cuts }}\right) .
$$

Conversely, if $h$ is nonnegative, lower semicontinuous and of bounded pointwise variation, then there exists a sequence $\left\{h_{n}\right\}$ of Lipschitz functions such that $0 \leq h_{n} \leq h, \mathbb{R}^{2} \backslash \Omega_{h_{n}}$ converges in the Hausdorff metric to $\mathbb{R}^{2} \backslash \Omega_{h}$, and

$$
\lim _{n \rightarrow+\infty} \int_{0}^{1} \sqrt{a_{n}+\left|h_{n}^{\prime}\right|^{2}} d x=\int_{0}^{1}\left|h^{\prime}\right|+2 \mathcal{H}^{1}\left(\Gamma_{\text {cuts }}\right)
$$

for every sequence $\left\{a_{n}\right\} \subset \mathbb{R}^{+}$with $a_{n} \rightarrow 0$.

By Proposition 2.3, following the argument of [20, Theorem 2.8], we obtain the following result. 
Proposition 2.4. For every nonnegative, lower semicontinuous function $h$ of bounded pointwise variation, and for every $u \in W^{1, p}\left(\Omega_{h} ; \mathbb{R}^{2}\right)$, there exist a sequence $\left\{h_{n}\right\}$ of nonnegative Lipschitz functions with $h_{n}(0)=h_{n}(1)=0$ and $\int_{0}^{1} h_{n} d x=\int_{0}^{1} h d x$ such that

$$
\lim _{n \rightarrow+\infty} \int_{0}^{1} \sqrt{a_{n}+\left|h_{n}^{\prime}\right|^{2}} d x=\int_{0}^{1}\left|h^{\prime}\right|+2 \mathcal{H}^{1}\left(\Gamma_{\text {cuts }}\right)
$$

for every sequence $\left\{a_{n}\right\} \subset \mathbb{R}^{+}$with $a_{n} \rightarrow 0$, and a sequence $\left\{u_{n}\right\} \subset W^{1, p}\left(\Omega_{h_{n}} ; \mathbb{R}^{2}\right)$ which converges strongly (locally) to $u$ in $W^{1, p}$.

Proof. By Proposition 2.3, there exists a sequence $\left\{h_{n}\right\}$ of Lipschitz functions such that $h_{n} \leq h$ and

$$
\lim _{n \rightarrow+\infty} \int_{0}^{1} \sqrt{a_{n}+\left|h_{n}^{\prime}\right|^{2}} d x=\int_{0}^{1}\left|h^{\prime}\right|+2 \mathcal{H}^{1}\left(\Gamma_{\text {cuts }}\right)
$$

for every sequence $a_{n} \rightarrow 0$. Set

$$
\varepsilon_{n}:=\int_{0}^{1}\left(h(x)-h_{n}(x)\right) d x
$$

and $\tilde{h}_{n}:=h_{n}+2 \varepsilon_{n}$. For $a>0$ such that $\left\{y=\tilde{h}_{n}(x)\right\} \cap\{y=a x\} \neq \emptyset$, let $\left(x_{a}, a x_{a}\right)=$ $\left(x_{a}, \tilde{h}_{n}\left(x_{a}\right)\right)$ be the first point of the intersection between $\left\{y=\tilde{h}_{n}(x)\right\}$ and $\{y=a x\}$ (see Figure 2), i.e.,

$$
x_{a}:=\inf \left\{x \in(0,1): a x=\tilde{h}_{n}(x)\right\} .
$$

Choose $a>0$ such that $\left|\Omega_{\tilde{h}_{n}} \cap\left\{(x, y): 0 \leq x \leq x_{a}, y \geq a x\right\}\right|=\frac{\varepsilon_{n}}{2}$. Notice that since $\frac{\varepsilon_{n}}{2} \geq x_{a} \varepsilon_{n}$ (the area of the shaded region on the left of Figure 2 is larger than the area of the triangle of height $2 \varepsilon_{n}$ and width $x_{a}$ ), we have $x_{a} \leq \frac{1}{2}$. Similarly, define $b>0$ and $x_{b} \geq \frac{1}{2} \geq x_{a}$ such that $\left|\Omega_{\tilde{h}_{n}} \cap\left\{(x, y): x_{b} \leq x \leq 1, y \geq b(1-x)\right\}\right|=\frac{\varepsilon_{n}}{2}$. Then

$$
\bar{h}_{n}(x):= \begin{cases}a x & \text { if } 0 \leq x \leq x_{a}, \\ \tilde{h}_{n}(x) & \text { if } x_{a} \leq x \leq x_{b}, \\ b(1-x) & \text { if } x_{b} \leq x \leq 1\end{cases}
$$

and

$$
u_{n}(x):= \begin{cases}e_{0}(x, 0) & \text { if } 0 \leq y \leq 2 \varepsilon_{n}, \\ u\left(x, y-2 \varepsilon_{n}\right) & \text { if } y \geq 2 \varepsilon_{n},\end{cases}
$$

satisfy the required properties.

Remark 2.5. The Dirichlet boundary condition is often relaxed. Precisely, instead of restricting ourselves to Lipschitz functions $h$ with $h(0)=h(1)=0$, we can equivalently consider arbitrary Lipschitz functions $h$ if the surface energy term is replaced by

$$
\int_{0}^{1} \sqrt{1+\left|h^{\prime}\right|^{2}} d x+h(0)+h(1)
$$




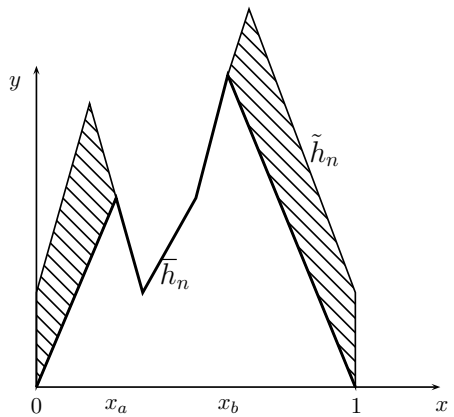

Figure 2: Construction of $\bar{h}_{n}$ from $\tilde{h}_{n}$ (see proof of Proposition 2.4)

This kind of relaxation is very classical for functionals with linear growth (see [28]).

Finally, we state an isoperimetric inequality which will be used throughout the paper.

Lemma 2.6. Let $h:[a, b] \rightarrow \mathbb{R}$ be a Lipschitz function on the segment $[a, b]$ with $h(a)=$ $h(b)=0$, and let $\int_{a}^{b} h(x) d x=: d$. Then

$$
\int_{a}^{b} \sqrt{1+\left|h^{\prime}\right|^{2}} d x \geq 2 \frac{d}{b-a}
$$

Proof. Let $\bar{x}$ be a point where $h$ attains its maximum on $[a, b]$ and set $\bar{h}:=h(\bar{x})$. Then

$$
\int_{a}^{b} \sqrt{1+\left|h^{\prime}\right|^{2}} d x \geq\left|\int_{a}^{\bar{x}} h^{\prime} d x\right|+\left|\int_{\bar{x}}^{b} h^{\prime} d x\right|=2 \bar{h} \geq 2 \frac{d}{b-a} .
$$

\subsection{The Strain Energy}

In this section we collect assumptions and basic properties of the strain energy term.

Definition 2.7. A Borel measurable function $W: \mathbb{R}^{2 \times 2} \rightarrow \mathbb{R}$ is quasiconvex if for every $A \in \mathbb{R}^{2 \times 2}$ and every bounded domain $\Omega \subset \mathbb{R}^{2}$

$$
W(A) \leq \frac{1}{|\Omega|} \int_{\Omega} W(A+\nabla \phi) d x d y \quad \text { for all } \quad \phi \in W_{0}^{1, \infty}\left(\Omega ; \mathbb{R}^{2}\right) .
$$

Remark 2.8. It is well known that in the definition of quasiconvexity one can equivalently suppose that (3) holds only for the unit cube $(0,1)^{2}$ (see [29]).

We assume that the energy density $W: \mathbb{R}^{2 \times 2} \rightarrow \mathbb{R}$ satisfies the following hypotheses:

(H1) $W \geq 0$;

(H2) $W$ is quasiconvex; 
(H3) there exist $\alpha>0$ and $1<p<\infty$ such that

$$
\alpha\left(|A|^{p}+1\right) \geq W(A) \geq \frac{1}{\alpha}\left(|A|^{p}-1\right) \quad \text { for all } A \in \mathbb{R}^{2 \times 2} .
$$

In case of geometrically linear elasticity, (H3) is replaced by the condition

(H3') there exist $\alpha>0$ and $1<p<\infty$ such that

$$
\alpha\left(\left|A+A^{T}\right|^{p}+1\right) \geq W(A) \geq \frac{1}{\alpha}\left(\left|A+A^{T}\right|^{p}-1\right) \quad \text { for all } A \in \mathbb{R}^{2 \times 2} .
$$

We note that we assume (H2) only to simplify notation. Indeed, the analysis goes through exactly in the same way provided that in the results the function $W$ is replaced by its quasiconvex envelope, i.e., the largest quasiconvex function which is below $W$. Hypothesis (H3) does not allow for potentials that blow-up when $\operatorname{det} y \rightarrow 0^{+}$, which corresponds to the noninterpenetration condition (see [8] for a discussion of this issue). By (H3) and a classical result of Marcellini (see [29, Lemma 5.2]), $W$ is locally $p$-Lipschitz continuous. Moreover, by (H3) and by density, the class of test functions in (3) can be enlarged to $W_{0}^{1, p}\left(\Omega ; \mathbb{R}^{2}\right)$.

We consider the recession function of $W$ at infinity defined by

$$
W^{\infty}(A):=\limsup _{t \rightarrow+\infty} \frac{W(t A)}{t^{p}} \text { for } A \in \mathbb{R}^{2 \times 2} .
$$

By definition, $W^{\infty}$ is $p$-homogeneous and satisfies the growth condition $\alpha|A|^{p} \geq W^{\infty}(A) \geq$ $\frac{1}{\alpha}|A|^{p}$, and similarly in the case of linear elasticity. Using the quasiconvexity of $W$ and the Fatou Lemma, we see that $W^{\infty}$ is quasiconvex, and therefore also locally $p$-Lipschitz continuous. The recession function arises naturally in relaxation and $\Gamma$-convergence problems for functionals with linear growth (see $[22,23]$ ). We will need the following additional assumption on the rate of convergence of $\frac{W(t A)}{t^{p}}$ to $W^{\infty}(A)$ (see [23]):

(H4) There exist $0<m<p, \gamma>0$ and $L>0$ such that for $t|A| \geq L$,

$$
\left|W^{\infty}(A)-\frac{W(t A)}{t^{p}}\right| \leq \gamma \frac{|A|^{p-m}}{t^{m}} .
$$

In the case of linear elasticity, this condition becomes:

(H4') There exist $0<m<p, \gamma>0$ and $L>0$ such that for $t\left|A+A^{T}\right| \geq L$,

$$
\left|W^{\infty}(A)-\frac{W(t A)}{t^{p}}\right| \leq \gamma \frac{\left|A+A^{T}\right|^{p-m}}{t^{m}} .
$$

Remark 2.9. For $p>1$ the functions $W(\cdot):=\operatorname{dist}^{p}\left(\cdot, S O_{2}\right)$ satisfy (H4). 
The main geometrical objects that will appear in the analysis are rectangles $[0, \ell] \times[0, L]$ for some $\ell, L>0$. We will use the following rescaling estimate.

Lemma 2.10. If $u \in W^{1, p}\left([0, \ell] \times[0, L] ; \mathbb{R}^{2}\right)$, with $u(x, 0)=e_{0}(x, 0)$, then

$$
\int_{[0, \ell] \times[0, L]} W(\nabla u) d x d y \geq e_{0}^{p} \ell^{2} \min _{v(x, 0)=(x, 0)} \int_{[0,1] \times[0, L / \ell]} \frac{W\left(e_{0} \nabla v\right)}{e_{0}^{p}} d x d y
$$

where here, and in the sequel, the minimum is taken among all $v \in W^{1, p}\left([0,1] \times[0, L / \ell] ; \mathbb{R}^{2}\right)$ that satisfy the boundary condition $v(x, 0)=(x, 0)$.

Proof. For $u \in W^{1, p}\left([0, \ell] \times[0, L] ; \mathbb{R}^{2}\right)$ set $\tilde{u}(x, y):=\frac{1}{\ell} u(\ell x, \ell y)$. Then $\tilde{u} \in W^{1, p}\left([0,1] \times[0, L / \ell] ; \mathbb{R}^{2}\right)$ with $\nabla \tilde{u}(x, y)=\nabla u(\ell x, \ell y)$, and thus

$$
\begin{aligned}
\min _{u(x, 0)=e_{0}(x, 0)} \int_{[0, \ell] \times[0, L]} W(\nabla u) d x d y & =\ell^{2} \min _{\tilde{u}(x, 0)=e_{0}(x, 0)} \int_{[0,1] \times[0, L / \ell]} W(\nabla \tilde{u}) d x d y \\
& =\ell^{2} \min _{v(x, 0)=(x, 0)} \int_{[0,1] \times[0, L / \ell]} W\left(e_{0} \nabla v\right) d x d y .
\end{aligned}
$$

Notice that for every $e_{0}$, the function

$$
\phi\left(t, e_{0}\right):=\min _{v(x, 0)=(x, 0)} \int_{[0,1] \times[0, t]} \frac{W\left(e_{0} \nabla v\right)}{e_{0}^{p}} d x d y
$$

is non-decreasing in $t$, and bounded above by some constant independent of $e_{0}$ (take any smooth function with bounded support and use (H3) or (H3')).

Remark 2.11. We recall that Korn-type inequalities or related rigidity results in the geometrically non-linear setting, degenerate if the Lipschitz constants of the domain blow-up, or if $L \rightarrow+\infty$ in strips of the form $[0, \ell] \times[0, L[$ (see $[36,24])$.

The following lemma describes the behavior of the elastic energy for small thickness of the film. It can be seen as a special case of a dimension reduction argument (see $[37,5])$.

Lemma 2.12. There holds

$$
\lim _{\varepsilon \rightarrow 0^{+}} \min _{v(x, 0)=(x, 0)} \frac{1}{\varepsilon} \int_{[0,1] \times[0, \varepsilon]}|\nabla v|^{p} d x d y=1,
$$

and similarly

$$
\lim _{\varepsilon \rightarrow 0^{+}} \min _{v(x, 0)=(x, 0)} \frac{1}{\varepsilon} \int_{[0,1] \times[0, \varepsilon]}|E(v)|^{p} d x d y=1,
$$

where $E(v)=\frac{\nabla v+\nabla^{T} v}{2}$. 
Proof. We prove only (5) since the proof of (6) is very similar. The inequality

$$
\min _{v(x, 0)=(x, 0)} \frac{1}{\varepsilon} \int_{[0,1] \times[0, \varepsilon]}|\nabla v|^{p} d x d y \leq 1
$$

being easily obtained by taking $v(x, y)=(x, 0)$, we are left to prove the reverse inequality.

In order to work on an $\varepsilon$-independent domain, we make a classical change of variables (see [15, Chapter 1, Section 1.3]), i.e., for $v=\left(v_{1}, v_{2}\right) \in W^{1, p}\left([0,1] \times[0, \varepsilon] ; \mathbb{R}^{2}\right)$ we set $w(x, y):=\left(v_{1}(x, \varepsilon y), \varepsilon v_{2}(x, \varepsilon y)\right) \in W^{1, p}\left([0,1]^{2} ; \mathbb{R}^{2}\right)$. Notice that if $v(x, 0)=(x, 0)$, then also $w(x, 0)=(x, 0)$. The rescaled functional to be considered then reads

$$
J_{\varepsilon}(w):=\int_{[0,1]^{2}}\left|\left(\begin{array}{cc}
\frac{\partial w_{1}}{\partial x} & \frac{1}{\varepsilon} \frac{\partial w_{1}}{\partial y} \\
\frac{1}{\varepsilon} \frac{\partial w_{2}}{\partial x} & \frac{1}{\varepsilon^{2}} \frac{\partial w_{2}}{\partial y}
\end{array}\right)\right|^{p} d x d y .
$$

If $w^{\varepsilon}$ is a minimizer of $J_{\varepsilon}$ subject to the condition $w^{\varepsilon}(x, 0)=(x, 0)$ then $\left\|w^{\varepsilon}\right\|_{W^{1, p}}$ is bounded above uniformly in $\varepsilon$. In particular, up to a subsequence, $\left\{w^{\varepsilon}\right\}$ converges weakly in $W^{1, p}$ to a function $w$ with $\frac{\partial w_{1}}{\partial y}=\frac{\partial w_{2}}{\partial x}=\frac{\partial w_{2}}{\partial y}=0$. Furthermore, $w(x, 0)=(x, 0)$ since for $p>1$ the space $W^{1, p}\left([0,1]^{2} ; \mathbb{R}^{2}\right)$ is compactly embedded in some $L^{q}\left([0,1]^{2} ; \mathbb{R}^{2}\right)$ (see $[1$, Theorem 6.3]). Thus

$$
\liminf _{\varepsilon \rightarrow 0^{+}} J_{\varepsilon}\left(w^{\varepsilon}\right) \geq \int_{[0,1]^{2}}\left|\frac{\partial w_{1}}{\partial x}\right|^{p} d x d y=1
$$

We next examine the scaling of the elastic energy when the thickness of the film tends to infinity.

Lemma 2.13. Let $W$ satisfy (H2), (H3) and (H4). If $f: \mathbb{R}^{+} \rightarrow \mathbb{R}^{+}$is such that $f(t) \rightarrow+\infty$ for $t \rightarrow+\infty$, then

$$
\lim _{e_{0} \rightarrow+\infty} \min _{v(x, 0)=(x, 0)} \int_{[0,1] \times\left[0, f\left(e_{0}\right)\right]} \frac{W\left(e_{0} \nabla v\right)}{e_{0}^{p}} d x d y=\min _{v(x, 0)=(x, 0)} \int_{[0,1] \times[0,+\infty)} W^{\infty}(\nabla v) d x d y .
$$

Similarly, let $W$ satisfy (H2), (H3') and (H4'). If $f: \mathbb{R}^{+} \rightarrow \mathbb{R}^{+}$is such that $f(t) \rightarrow+\infty$ for $t \rightarrow+\infty$, then

$$
\lim _{e_{0} \rightarrow+\infty} \min _{v(x, 0)=(x, 0)} \int_{[0,1] \times\left[0, f\left(e_{0}\right)\right]} \frac{W\left(e_{0} E(v)\right)}{e_{0}^{p}} d x d y=\min _{v(x, 0)=(x, 0)} \int_{[0,1] \times[0,+\infty)} W^{\infty}(E(v)) d x d y .
$$

Proof. As before, we only prove (7) since (8) can be very similarly obtained. Set

$$
G_{e_{0}}(v):=\int_{[0,1] \times\left[0, f\left(e_{0}\right)\right]} \frac{W\left(e_{0} \nabla v\right)}{e_{0}^{p}} d x d y \quad \text { and } \quad G(v):=\int_{[0,1] \times[0,+\infty)} W^{\infty}(\nabla v) d x d y .
$$


By Theorem 2.2, it suffices to show that $G_{e_{0}} \stackrel{\Gamma}{\rightarrow} G$ with respect to the (sequential) weak topology of $W_{\text {loc }}^{1, p}\left([0,1] \times[0,+\infty) ; \mathbb{R}^{2}\right)$ as $e_{0} \rightarrow+\infty$. Consider first $\left\{v_{e_{0}}\right\}$ such that $v_{e_{0}}(x, 0)=$ $(x, 0)$ and $\sup G_{e_{0}}\left(v_{e_{0}}\right)<+\infty$. Then by (H3), for every $M>0$ the functions $v_{e_{0}}$ are uniformly bounded in $W^{1, p}\left([0,1] \times[0, M] ; \mathbb{R}^{2}\right)$, and thus there exists a subsequence converging weakly in $W_{\text {loc }}^{1, p}$ to a function $v$.

We now show that for $M>0$ fixed

$$
\liminf _{e_{0} \rightarrow+\infty} \int_{[0,1] \times[0, M]} \frac{W\left(e_{0} \nabla v_{e_{0}}\right)}{e_{0}^{p}} d x d y \geq \int_{[0,1] \times[0, M]} W^{\infty}(\nabla v) d x d y .
$$

The proof of (9) is an adaptation of the semicontinuity proof for quasiconvex functionals (see $[29$, Thm. 5.4]), and we sketch it only for the reader's convenience. Set $R:=[0,1] \times[0, M]$. For simplicity of notation, let $\mathbf{x}:=(x, y)^{T}$.

Step 1. If $v$ is affine, i.e., $v=A \mathbf{x}+b$ for some $A \in \mathbb{R}^{2 \times 2}$ and some $b \in \mathbb{R}^{2}$, and $v_{e_{0}}=v$ on $\partial R$, then $v_{e_{0}}=v+\phi_{e_{0}}$ for some $\phi_{e_{0}} \in W_{0}^{1, p}\left(R ; \mathbb{R}^{2}\right)$, and by (3) and (H4), for $e_{0}|A| \geq L$ or $A=0$ we have

$$
\int_{R} \frac{W\left(e_{0} \nabla v_{e_{0}}\right)}{e_{0}^{p}} d x d y \geq M \frac{W\left(e_{0} A\right)}{e_{0}^{p}} \geq M\left(W^{\infty}(A)-\gamma \frac{|A|^{p-m}}{e_{0}^{m}}\right),
$$

and (9) follows as $e_{0} \rightarrow+\infty$ by quasiconvexity of $W^{\infty}$.

Step 2. If $v$ is affine but $v_{e_{0}} \neq v$ on $\partial R$, then we modify $v_{e_{0}}$ near $\partial R$. Precisely, for $R_{0} \Subset R$, $K \in \mathbb{N}$ and $i=1, \ldots, K$, consider the sets

$$
R_{i}:=\left\{\mathrm{x} \in R: \operatorname{dist}\left(\mathbf{x}, R_{0}\right)<\frac{i}{K} \rho\right\}
$$

where $\rho:=\frac{1}{2} \operatorname{dist}\left(R_{0}, \partial R\right)$, and introduce cut-off functions $\psi_{i} \in C_{0}^{1}\left(R_{i}\right)$ with

$$
0 \leq \psi_{i} \leq 1, \quad \psi_{i}=1 \text { in } \quad R_{i-1}, \quad \text { and } \quad\left|\nabla \psi_{i}\right| \leq \frac{2(K+1)}{\rho} .
$$

Set $w_{e_{0}}^{i}:=v+\psi_{i}\left(v_{e_{0}}-v\right)$. Notice that

$$
\left|\nabla w_{e_{0}}^{i}\right|^{p} \leq C\left(|\nabla v|^{p}+\left|\nabla v_{e_{0}}\right|^{p}+\frac{(K+1)^{p}}{\rho^{p}}\left|v_{e_{0}}-v\right|^{p}\right) .
$$

Since $w_{e_{0}}^{i}=v$ on $\partial R$, by (10) we have

$$
\int_{R} \frac{W\left(e_{0} \nabla w_{e_{0}}^{i}\right)}{e_{0}^{p}} d x d y \geq \int_{R} W^{\infty}(\nabla v) d x d y-M \gamma \frac{|A|^{p-m}}{e_{0}^{m}} .
$$


Therefore,

$$
\begin{aligned}
\int_{R} W^{\infty}(\nabla v) d x d y-M \gamma \frac{|A|^{p-m}}{e_{0}^{m}} \leq & \int_{R \backslash R_{i}} \frac{W\left(e_{0} \nabla v\right)}{e_{0}^{p}} d x d y+\int_{R_{i} \backslash R_{i-1}} \frac{W\left(e_{0} \nabla w_{e_{0}}^{i}\right)}{e_{0}^{p}} d x d y \\
& +\int_{R_{i-1}} \frac{W\left(e_{0} \nabla v_{e_{0}}\right)}{e_{0}^{p}} d x d y \\
\leq & \int_{R \backslash R_{0}} W^{\infty}(\nabla v) d x d y+M \gamma \frac{|A|^{p-m}}{e_{0}^{m}} \\
& +\int_{R_{i} \backslash R_{i-1}} \frac{W\left(e_{0} \nabla w_{e_{0}}^{i}\right)}{e_{0}^{p}} d x d y+\int_{R} \frac{W\left(e_{0} \nabla v_{e_{0}}\right)}{e_{0}^{p}} d x d y .
\end{aligned}
$$

Since $v_{e_{0}} \rightarrow v$ weakly in $W^{1, p}(R)$, using (H3) and (11) we can bound the penultimate integral in the above inequality. Summing the inequalities over $i$, dividing by $K$ and letting $e_{0} \rightarrow+\infty$, we find that for some $C>0$

$$
\int_{R_{0}} W^{\infty}(\nabla v) d x d y \leq \liminf _{e_{0} \rightarrow+\infty} \int_{R} \frac{W\left(e_{0} \nabla v_{e_{0}}\right)}{e_{0}^{p}} d x d y+\frac{C}{K}
$$

which for $K \rightarrow+\infty$ and $R_{0} \rightarrow R$ implies (9).

Step 3. Consider then a countable family of squares $Q_{i}$ with pairwise disjoint interiors that cover $R$. We apply Step 2 in each square to the functions $\left\langle z_{i}, \cdot\right\rangle$, where

$$
\begin{gathered}
z_{i}:=\frac{1}{\left|Q_{i}\right|} \int_{Q_{i}} \nabla v d x d y \\
\liminf _{e_{0} \rightarrow+\infty} \int_{Q_{i}} \frac{W\left(e_{0} \nabla w_{e_{0}}^{i}\right)}{e_{0}^{p}} d x d y \geq \int_{Q_{i}} W^{\infty}\left(z_{i}\right) d x d y .
\end{gathered}
$$

Let $\bar{Z}$ be the function that in every cube $Q_{i}$ is equal to the constant $z_{i}$. If the diameters of the squares tend uniformly to zero then $\bar{Z}$ tends to $D v$ in $L^{p}(R)$ from which we get that for every $\varepsilon>0$ there is a covering $Q_{i}$ such that

$$
\begin{array}{r}
\left|\int_{R} \frac{W\left(e_{0} \nabla v_{e_{0}}\right)}{e_{0}^{p}} d x d y-\sum_{i} \int_{Q_{i}} \frac{W\left(e_{0} \nabla w_{e_{0}}^{i}\right)}{e_{0}^{p}} d x d y\right| \leq C \varepsilon \\
\text { and } \\
\left|\int_{R} W^{\infty}(\nabla v) d x d y-\sum_{i} \int_{Q_{i}} W^{\infty}\left(z_{i}\right)\right| \leq C \varepsilon,
\end{array}
$$

and (9) follows. In conclusion, for every $M>0$,

$$
\liminf _{e_{0} \rightarrow+\infty} G_{e_{0}}\left(v_{e_{0}}\right) \geq \liminf _{e_{0} \rightarrow+\infty} \int_{[0,1] \times[0, M]} \frac{W\left(e_{0} \nabla v_{e_{0}}\right)}{e_{0}^{p}} d x d y \geq \int_{[0,1] \times[0, M]} W^{\infty}(\nabla v) d x d y
$$


We let $M \rightarrow+\infty$ to get $\liminf G_{e_{0}}\left(v_{e_{0}}\right) \geq G(v)$.

On the other hand, if $v \in W^{1, p}\left([0,1] \times[0,+\infty) ; \mathbb{R}^{2}\right)$, then $v$ can be approximated in energy by functions with compact support and for these functions, using the growth conditions (H3) and the reverse Fatou Lemma, we conclude that

$$
\limsup _{e_{0} \rightarrow+\infty} G_{e_{0}}(v) \leq G(v) .
$$

Define

$$
C_{W}:=\min _{v(x, 0)=(x, 0)} \int_{[0,1] \times[0,+\infty)} W^{\infty}(\nabla v) d x d y .
$$

Remark 2.14. If $W(\cdot)=|\cdot|^{2}$, one obtains by Fourier methods that (see [17, Appendix])

$$
C_{W}=\frac{1}{\pi^{3}} \sum_{k>0} \frac{1}{k^{3}}\left(1+(-1)^{k+1}\right)^{2} .
$$

\section{Scaling law}

In this section we consider the qualitative behavior of the minimal energy in terms of its scaling regimes.

Theorem 3.1. Suppose that $W$ satisfies (H1) and (H3), or (H1) and (H3'). Then there are constants $C_{1}, C_{2}>0$ such that, for all $d, e_{0}>0$,

$$
C_{1} \max \left\{1, d, e_{0}^{p / 3} d^{2 / 3}\right\} \leq \inf F_{d, e_{0}} \leq C_{2} \max \left\{1, d, e_{0}^{p / 3} d^{2 / 3}\right\} .
$$

Proof. We give the proof only for the nonlinear elasticity since the geometrically linear case can be treated analogously.

\section{Upper bound.}

For $\ell \leq 1$ and $0<\delta \ll \frac{\ell}{2}$ set $\tilde{h}:=\frac{2 d}{2 \ell-\delta}$, and consider (see Figure $3(\mathrm{a})$ )

$$
h(x):= \begin{cases}\frac{2 \tilde{h}}{\delta} x & \text { if } 0 \leq x \leq \frac{\delta}{2}, \\ \tilde{h} & \text { if } \frac{\delta}{2} \leq x \leq \ell-\frac{\delta}{2} \\ -\frac{2 \tilde{h}}{\delta} x+\frac{2 \tilde{h}}{\delta} \ell & \text { if } \ell-\frac{\delta}{2} \leq x \leq \ell \\ 0 & \text { if } \ell \leq x \leq 1\end{cases}
$$

Let $u$ be the restriction to $\Omega_{h}$ of $\tilde{u}: I \times[0, \infty) \rightarrow \mathbb{R}$ given by

$$
\tilde{u}(x, y)= \begin{cases}\left(e_{0} x\left(1-\frac{1}{\ell} y\right), 0\right) & \text { if } 0 \leq y \leq \ell \\ 0 & \text { else }\end{cases}
$$




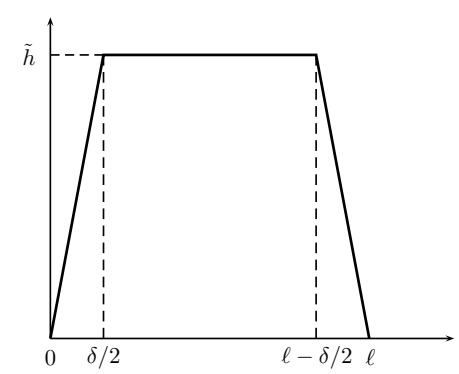

(a) Upper bound construction

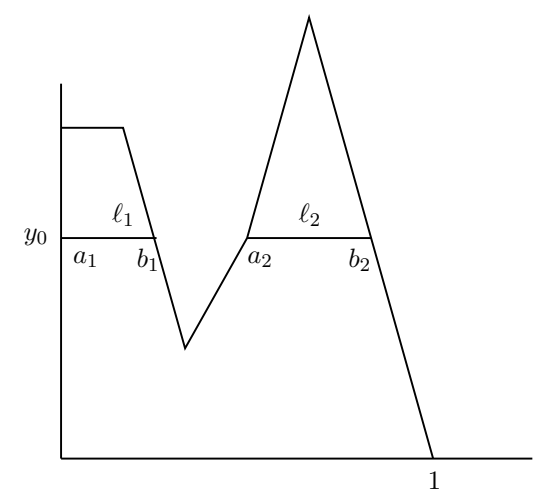

(b) Geometry for the lower bound

Figure 3: Constructions in the proof of Theorem 3.1

Then

$$
\begin{aligned}
F_{d, e_{0}}(u, h) & \leq \int_{\Omega_{h}} W(\nabla u) d x d y+1+\frac{4 d}{2 \ell-\delta} \\
& \leq C e_{0}^{p} \int_{0}^{\ell} \int_{0}^{\ell}\left(\left(1-\frac{1}{\ell} y\right)^{p}+\left(\frac{1}{\ell} x\right)^{p}\right) d x d y+\alpha d+1+\frac{4 d}{2 \ell-\delta} \\
& \leq C\left(e_{0}^{p} \ell^{2}+1+d+\frac{d}{\ell}\right)
\end{aligned}
$$

If $e_{0}^{p} \leq d$, then we choose $\ell:=1$, and if $e_{0}^{p} \geq d$ we choose $\ell:=d^{1 / 3} e_{0}^{-p / 3} \leq 1$. It follows that

$$
e_{0}^{p} \ell^{2}+1+d+\frac{d}{\ell} \leq C \max \left\{1, d, e_{0}^{p / 3} d^{2 / 3}\right\}
$$

and this completes the proof the of the upper bound.

Lower bound: By definition of the surface energy, $F_{d, e_{0}}(u, h) \geq 1$, and by Lemma 2.6 $F_{d, e_{0}}(u, h) \geq 2 d$. It remains to show that $F_{d, e_{0}}(u, h) \geq C e_{0}^{p / 3} d^{2 / 3}$ if $e_{0}^{p / 3} d^{2 / 3} \geq C \max \{1, d\}$ for some fixed constant $C>0$.

Let $y_{0}:=\frac{d}{\sqrt{e_{0}^{p / 3} d^{2 / 3}}}, \ell:=\mathcal{H}^{1}\left(\Omega_{h} \cap\left(I \times\left\{y_{0}\right\}\right)\right)$ and $I_{\ell}:=\Omega_{h} \cap\left(I \times\left\{y_{0}\right\}\right)$. Denote by $\left[a_{i}, b_{i}\right]$ the countably many (pairwise disjoint) maximal connected components of $I_{\ell}$ (see Figure 3(b)), i.e., $I_{\ell}=\cup_{i=1}^{+\infty}\left[a_{i}, b_{i}\right]$, and for $i \in \mathbb{N}$, set $\ell_{i}:=b_{i}-a_{i}$ and $d_{i}:=\left|\Omega_{h} \cap\left(\left[a_{i}, b_{i}\right] \times\left[y_{0},+\infty\right)\right)\right|$. Then $\sum_{i=1}^{+\infty} \ell_{i}=\ell$ and $\sum_{i=1}^{+\infty} d_{i} \geq d-y_{0} \geq C d$. 
If $\ell_{i} \leq\left(\frac{d}{e_{0}^{p}}\right)^{1 / 3}$ for all $i \in \mathbb{N}$, then by (2) applied to $h-y_{0}$ we obtain

$$
\begin{aligned}
F_{d, e_{0}}(u, h) & \geq \sum_{i=1}^{+\infty} \int_{a_{i}}^{b_{i}} \sqrt{1+\left|h^{\prime}\right|^{2}} d x \\
& \geq \sum_{i=1}^{+\infty} \frac{d_{i}}{\ell_{i}} \geq\left(\frac{e_{0}^{p}}{d}\right)^{1 / 3} \sum_{i=1}^{+\infty} d_{i} \\
& \geq C e_{0}^{p / 3} d^{2 / 3} .
\end{aligned}
$$

Otherwise, the largest connected component of $I_{\ell}$, say $I_{\ell_{1}}$, satisfies $\ell_{1} \geq\left(\frac{d}{e_{0}^{p}}\right)^{1 / 3}$. By Lemma 2.12 there exists a small constant $\varepsilon_{0}<1$ such that for $\varepsilon \leq \varepsilon_{0}$,

$$
\min _{u(x, 0)=(x, 0)} \frac{1}{\varepsilon} \int_{[0,1] \times[0, \varepsilon]}|\nabla u|^{p} d x d y \geq \frac{1}{2} .
$$

Since $\varepsilon_{0}>0$ is a universal constant, and since $e_{0}^{p / 3} d^{2 / 3} \geq C \max \{1, d\}$, we may, without loss of generality, by changing $C$, assume that $e_{0}^{p / 2} \geq \frac{4}{\varepsilon_{0}}$. If $\left(\frac{d}{e_{0}^{p}}\right)^{1 / 3} \leq \ell_{1} \leq \frac{y_{0}}{\varepsilon_{0}}$, then, by (4) and (H3),

$$
\begin{aligned}
F_{d, e_{0}}(u, h) & \geq \int_{\left[a_{1}, b_{1}\right] \times\left[0, y_{0}\right]} W(\nabla u) d x d y \\
& \geq \frac{1}{\alpha}\left(\frac{d}{e_{0}^{p}}\right)^{2 / 3} e_{0}^{p} \min _{v(x, 0)=(x, 0)} \int_{[0,1] \times\left[0, \varepsilon_{0}\right]}|\nabla v|^{p} d x d y-\frac{1}{\alpha} \ell_{1} y_{0} \\
& \geq \frac{\varepsilon_{0}}{2 \alpha} e_{0}^{p / 3} d^{2 / 3}-\frac{1}{\alpha} y_{0} \\
& \geq \frac{\varepsilon_{0}}{2 \alpha} e_{0}^{p / 3} d^{2 / 3}-\frac{1}{\alpha} \frac{d}{\sqrt{e_{0}^{p / 3} d^{2 / 3}}} \\
& \geq \frac{\varepsilon_{0}}{4 \alpha} e_{0}^{p / 3} d^{2 / 3},
\end{aligned}
$$

where we used that $\frac{d}{\sqrt{e_{0}^{p / 3} d^{2 / 3}}} \leq \frac{\varepsilon_{0}}{4} e_{0}^{p / 3} d^{2 / 3}$, since $e_{0}^{p / 2} \geq \frac{4}{\varepsilon_{0}}$.

Otherwise, if $\ell_{1} \geq y_{0} / \varepsilon_{0}$, then, by (4), (H3), (5), and since $\ell_{1} y_{0}=\frac{d}{\left(e_{0}^{p / 3} d^{2 / 3}\right)^{1 / 2}} \ell_{1} \leq e_{0}^{p / 3} d^{2 / 3}$,

$$
\begin{aligned}
F_{d, e_{0}}(u, h) & \geq \frac{1}{\alpha} \ell_{1}^{2} e_{0}^{p} \min _{v(x, 0)=(x, 0)} \int_{[0,1] \times\left[0, y_{0} / \ell_{1}\right]}|\nabla v|^{p} d x d y-\frac{1}{\alpha} \ell_{1} y_{0} \\
& \geq \frac{1}{2 \alpha} \ell_{1}^{2} e_{0}^{p} \frac{y_{0}}{\ell_{1}}-\frac{1}{\alpha} e_{0}^{p / 3} d^{2 / 3} \\
& \geq \frac{1}{2 \varepsilon_{0} \alpha}\left(e_{0}^{p / 3} d^{2 / 3}\right)^{2}-\frac{1}{\alpha} e_{0}^{p / 3} d^{2 / 3} \geq c e_{0}^{p / 3} d^{2 / 3}
\end{aligned}
$$

where the last inequality holds since $e_{0}^{p / 3} d^{2 / 3}>C$ for $C$ large enough. 


\section{Reduced models}

To study the asymptotic behavior of $F_{d, e_{0}}$ in the various regimes determined in Theorem 3.1, we set $\widetilde{h}:=h / d, \Omega_{\tilde{h}}:=\left\{(x, y):(x, d y) \in \Omega_{h}\right\}$, and $\widetilde{u}(x, y)=u(x, d y)$. Dropping the tildes, the energy now reads

$$
F_{d, e_{0}}(u, h)=d\left[\int_{\Omega_{h}} W\left(\frac{\partial u}{\partial x}, \frac{1}{d} \frac{\partial u}{\partial y}\right) d x d y+\int_{0}^{1} \sqrt{\frac{1}{d^{2}}+\left|h^{\prime}\right|^{2}} d x\right]
$$

for $(u, h)$ such that $h(0)=h(1)=0, \int_{0}^{1} h d x=1$, and $u \in W^{1, p}\left(\Omega_{h}\right)$ with $u(x, 0)=e_{0}(x, 0)$.

In the regime in which $F_{d, e_{0}} \simeq 1$, the limiting functional is constant (see Proposition 4.1), while for $F_{d, e_{0}} \simeq d$, the main contribution to the reduced energy comes from the surface part (see Proposition 4.2), and the flat configuration is optimal. Notice, however, that as proven in [26], for large but finite mismatch $e_{0}>0$ and for volume $d$ large enough, the flat configuration is never a global minimizer of the energy. We believe that this is a higher order effect that cannot be captured by our first order analysis. In the transition regime, in which $d \simeq e_{0}^{p} \rightarrow+\infty$, elastic and surface energy compete (see Proposition 4.3), and the minimizer of the reduced model is either the flat configuration or an island, depending on the elastic properties of the material, in particular on the constant $C_{W}$ in (12). Finally, in the regime in which $F_{d, e_{0}} \simeq e_{0}^{p / 3} d^{2 / 3}$, the elastic energy dominates. By a corresponding compactness result (see Proposition 4.7), we conclude that in this regime the formation of islands is energetically favored.

\subsection{The trivial regime $F_{d, e_{0}} \simeq 1$}

If $d \rightarrow 0$ and $e_{0}^{p}=o\left(\frac{1}{d^{2}}\right)$, then $F_{d, e_{0}} \simeq 1$, and the limit functional is a constant. In this regime, the non-horizontal parts of the surface and the elastic part of the energy do not contribute significantly. Given an absolutely continuous probability measure $\mu:=h d x$, let

$$
G_{d, e_{0}}(\mu):=\min _{u(x, 0)=e_{0}(x, 0)} F_{d, e_{0}}(u, h) .
$$

Proposition 4.1. Suppose that $W$ satisfies (H1) and (H3), $d \rightarrow 0$ and $e_{0}^{p}=o\left(\frac{1}{d^{2}}\right)$, then $\left\{G_{d, e_{0}}\right\} \Gamma$-converges with respect to the weak-* convergence of measures to the constant functional equal to 1 on probability measures.

Proof. The compactness follows from the weak-* compactness of sequences of probability measures on compact sets. Since the energy is always bounded from below by 1 , the lower bound is immediate. For the upper bound, by density of finite sums of Dirac masses in the set of probability measures, it is enough to assume that $\mu=\sum_{i=1}^{N} d_{i} \delta_{c_{i}}$, and we can further assume that for every $i=1, . ., N, c_{i} \notin\{0 ; 1\}$. If $\frac{d}{e_{0}^{p}} \rightarrow 0$, then we let $\ell_{i}:=\left(\frac{d d_{i}}{e_{0}^{p}}\right)^{1 / 3}$ and 
$h_{i}:=\frac{d_{i}}{\ell_{i}}$. We finally let $h_{d, e_{0}}$ be a Lipschitz approximation of $\sum_{i=1}^{N} h_{i} \chi_{\left[c_{i}-\frac{\ell_{i}}{2}, c_{i}+\frac{\ell_{i}}{2}\right]}$ and $u_{d, e_{0}}$ be the minimizer of the elastic energy in $\Omega_{h_{d, e_{0}}}$ so that

$$
F_{d, e_{0}}\left(u_{d, e_{0}}, h_{d, e_{0}}\right) \leq 1+2 \sum_{i=1}^{N} \frac{d d_{i}}{\ell_{i}}+C \sum_{i=1}^{N} e_{0}^{p} \ell_{i}^{2} \leq 1+C e_{0}^{p / 3} d^{2 / 3}
$$

from which the upper bound follows. If $d \geq C e_{0}^{p}$, we then replace in the previous construction $\ell_{i}$ by $\ell_{i}:=d^{1 / 2}$ from which we similarly obtain

$$
F_{d, e_{0}}\left(u_{d, e_{0}}, h_{d, e_{0}}\right) \leq 1+2 \sum_{i=1}^{N} \frac{d d_{i}}{\ell_{i}}+C \sum_{i=1}^{N} e_{0}^{p} \ell_{i}^{2} \leq 1+2 d^{1 / 2}+C e_{0}^{p} d^{2} .
$$

\subsection{The surface dominant regime $F_{d, e_{0}} \simeq d$}

If $d \rightarrow+\infty$ and $e_{0}^{p}=o(d)$, then $F_{d, e_{0}} \simeq d$. Hence, we rescale the energy by $d$, and consider

$$
F_{d}(u, h):=\int_{\Omega_{h}} W\left(\frac{\partial u}{\partial x}, \frac{1}{d} \frac{\partial u}{\partial y}\right) d x d y+\int_{0}^{1} \sqrt{\frac{1}{d^{2}}+\left|h^{\prime}\right|^{2}} d x .
$$

In this regime, the surface energy dominates, and the limit functional turns out to be

$$
\bar{F}(h):=\int_{0}^{1}\left|h^{\prime}\right|+2 \mathcal{H}^{1}\left(\Gamma_{\text {cuts }}\right) .
$$

Proposition 4.2. Suppose that $W$ satisfies (H1) and (H3), and let $\left\{\left(u_{d}, h_{d}\right)\right\}$ be such that $\sup _{d} F_{d}\left(u_{d}, h_{d}\right)<+\infty$. Then there is a subsequence (not relabaled) such that the sets $\mathbb{R}^{2} \backslash \Omega_{h_{d}}$ converge to $\mathbb{R}^{2} \backslash \Omega_{h}$ in the Hausdorff metric, where $h(x):=\inf \left\{\liminf h_{d}\left(x_{d}\right): x_{d} \rightarrow x\right\}$ and

$$
\liminf _{d \rightarrow+\infty} F_{d}\left(u_{d}, h_{d}\right) \geq \bar{F}(h) .
$$

Further, for every nonnegative lower semicontinuous function $h$ with bounded pointwise variation and $\int_{0}^{1} h(x) d x=1$, there exists a sequence $\left\{\left(u_{d}, h_{d}\right)\right\}$ such that $h_{d}: I \rightarrow \mathbb{R}$ are non-negative Lipschitz functions with $\int_{0}^{1} h_{d}(x) d x=1, h_{d}(0)=h_{d}(1)=0$, and $u_{d} \in$ $W^{1, p}\left(\Omega_{h_{d}} ; \mathbb{R}^{2}\right), \mathbb{R}^{2} \backslash \Omega_{h_{d}}$ converge to $\mathbb{R}^{2} \backslash \Omega_{h}$, and

$$
\limsup _{d \rightarrow+\infty} F_{d}\left(u_{d}, h_{d}\right) \leq \bar{F}(h) .
$$

Proof. The compactness and the liminf inequality follow from Proposition 2.3. For the limsup inequality, we may assume that $h$ is Lipschitz continuous with $h(0)=h(1)=0$ (see Proposition 2.4). Choose $h_{d}:=h$ and

$$
u_{d}(x, y):= \begin{cases}e_{0}(x(1-d y), 0) & \text { if } y \leq \frac{1}{d} \\ 0 & \text { if } y \geq \frac{1}{d}\end{cases}
$$


Then by (H3),

$$
\begin{aligned}
\int_{\Omega_{h}} W\left(\frac{\partial u_{d}}{\partial x}, \frac{1}{d} \frac{\partial u_{d}}{\partial y}\right) d x d y & \leq \int_{0}^{1} \int_{0}^{1 / d} \alpha\left(e_{0}^{p}|(1-d y,-x)|^{p}+1\right) d y d x \\
& \leq \alpha\left(\frac{e_{0}^{p}}{d} \int_{0}^{1} \int_{0}^{1}|(1-y, x)|^{p} d x d y+\frac{1}{d}\right)
\end{aligned}
$$

and thus $\lim \sup _{d \rightarrow+\infty} \int_{\Omega_{h}} W\left(\frac{\partial u_{d}}{\partial x}, \frac{1}{d} \frac{\partial u_{d}}{\partial y}\right) d x d y=0$.

\subsection{The borderline case $d \simeq e_{0}^{p}$}

We now focus on the limit case $d \simeq e_{0}^{p} \rightarrow+\infty$. In this regime, elastic and surface energy compete and interplay in the limit. Rescaling the energy by $d$, we obtain

$$
F_{d}(u, h):=\int_{\Omega_{h}} W\left(\frac{\partial u}{\partial x}, \frac{1}{d} \frac{\partial u}{\partial y}\right) d x d y+\int_{0}^{1} \sqrt{\frac{1}{d^{2}}+\left|h^{\prime}\right|^{2}} d x .
$$

We first give a compactness and lower-bound result.

Proposition 4.3. Suppose that $W$ satisfies (H1)-(H4). If $\sup F_{d}\left(u_{d}, h_{d}\right)<+\infty$ then there is a subsequence with the following properties: $\left\{\mathbb{R}^{2} \backslash \Omega_{h_{d}}\right\}$ converges in the Hausdorff topology to $\mathbb{R}^{2} \backslash \Omega_{h}$, where $h(x):=\inf \left\{\liminf h_{d}\left(x_{d}\right): x_{d} \rightarrow x\right\}$. Denote the boundary layers by

$$
B L_{h}:=\left\{(x, y):(x, y) \in[0,1] \times \mathbb{R}^{+}, 0<h(x)\right\}
$$

and

$$
B L_{h}^{d}:=\left\{(x, y):(x, y) \in B L_{h}, y \leq d h_{d}(x)\right\} .
$$

Then $\left\{B L_{h}^{d}\right\}$ converges in the local Hausdorff topology to $B L_{h}$. Moreover, if $v_{d}: B L_{h}^{d} \rightarrow \mathbb{R}^{2}$ is defined by $v_{d}(x, y):=\frac{1}{e_{0}} u_{d}\left(x, \frac{y}{d}\right)$, then there exists $v \in W^{1, p}\left(B L_{h} ; \mathbb{R}^{2}\right)$ with $v(x, 0)=(x, 0)$, $v_{d} \chi_{B L_{h}^{d}} \rightarrow v$ locally weakly in $W_{\text {loc }}^{1, p}\left(B L_{h} ; \mathbb{R}^{2}\right)$, and

$$
\liminf _{d \rightarrow+\infty} F_{d}\left(u_{d}, h_{d}\right) \geq \int_{B L_{h}} W^{\infty}(\nabla v) d x d y+\int_{0}^{1}\left|h^{\prime}\right|+2 \mathcal{H}^{1}\left(\Gamma_{\text {cuts }}\right) .
$$

Proof. Since $\left|\Omega_{h_{d}}\right|=1$ and $\left\{\int_{0}^{1} \sqrt{\frac{1}{d^{2}}+\left|h_{d}^{\prime}\right|^{2}} d x\right\}$ is uniformly bounded, the compactness and lower-semicontinuity for the surface part follow from Proposition 2.3, and the convergence of $\left\{B L_{h}^{d}\right\}$ follows from the Hausdorff convergence of $\mathbb{R}^{2} \backslash \Omega_{h_{d}}$.

We now focus on the elastic energy. Changing variables $z=d y$ and dividing by $e_{0}$, we get

$$
\int_{B L_{h}^{d}} \frac{W\left(e_{0} \nabla v_{d}\right)}{e_{0}^{p}} d x d y \leq C
$$


which, by the $p$-growth condition (H3), implies that $\left\|\nabla v_{d}\right\|_{L^{p}\left(B L_{h}^{d} \cap\{y \leq M\}\right)}$ is bounded for every $M$. Hence, as in Lemma 2.12, $\left\|v_{d}\right\|_{L^{p}\left(B L_{h}^{d} \cap\{y \leq M\}\right)}$ is also bounded, and so there exists a subsequence that converges locally to $v \in W_{l o c}^{1, p}\left(B L_{h}\right)$ with $v(x, 0)=(x, 0)$. The liminf inequality follows from the weak convergence, as in Lemma 2.13.

We are now in position to prove the limsup inequality:

Proposition 4.4. Suppose $W$ satisfies (H1)-(H4). For every pair $(v, h)$ with $v \in W_{l o c}^{1, p}\left(B L_{h} ; \mathbb{R}^{2}\right)$, $v(x, 0)=(x, 0)$, and $h: I \rightarrow \mathbb{R}$ a nonnegative lower semicontinuous function of bounded pointwise variation with $\int_{0}^{1} h(x) d x=1$, there exists a sequence $\left\{u_{d}\right\} \subset W_{l o c}^{1, p}\left(\Omega_{h} ; \mathbb{R}^{2}\right)$ with $u_{d}(x, 0)=e_{0}(x, 0)$, such that $\frac{1}{e_{0}} u_{d}\left(x, \frac{y}{d}\right) \rightarrow v$ locally weakly in $W_{l o c}^{1, p}\left(B L_{h} ; \mathbb{R}^{2}\right)$ and

$$
\lim _{d \rightarrow+\infty} F_{d}\left(u_{d}, h\right)=\int_{B L_{h}} W^{\infty}(\nabla v) d x d y+\int_{0}^{1}\left|h^{\prime}\right|+2 \mathcal{H}^{1}\left(\Gamma_{\text {cuts }}\right) .
$$

Proof. We may assume that $h$ is Lipschitz continuous (see Proposition 2.4), and that $v$ has bounded support. Define the recovery sequence by $u_{d}(x, y):=e_{o} v(x, d y)$. Then $\frac{1}{e_{0}} u_{d}\left(x, \frac{y}{d}\right) \rightarrow$ $v$ locally weakly in $W_{l o c}^{1, p}\left(B L_{h} ; \mathbb{R}^{2}\right)$ and

$$
\lim _{d \rightarrow+\infty} F_{d}\left(u_{d}, h\right)=\int_{B L_{h}} W^{\infty}(\nabla v) d x d y+\int_{0}^{1}\left|h^{\prime}\right|
$$

We now characterize the minimizers of the limiting functional.

Proposition 4.5. Assume that $W$ satisfies (H1)-(H4), and let $C_{W}$ be as in (12). If $C_{W} \geq 1$, then the minimum of

$$
\int_{B L_{h}} W^{\infty}(\nabla v) d x d y+\int_{0}^{1}\left|h^{\prime}\right|+2 \mathcal{H}^{1}\left(\Gamma_{\text {cuts }}\right)
$$

is attained for $h=\frac{1}{\ell_{\min }} \chi_{\left[0, \ell_{\min }\right]}$ with $\ell_{\min }=\left(\frac{1}{C_{W}}\right)^{1 / 3}$, and $v$ the minimizer of the elastic energy in the corresponding boundary layer $B L_{h}$. If $C_{W}<1$, then the flat configuration is minimizing.

Proof. Note first that $h$ is constant on each connected component of $\{h \neq 0\}$. Indeed, given an interval $[a, b]$ and a volume $d$, the rectangle is the shape with least vertical perimeter (i.e., minimal $\left.\int_{a}^{b}\left|h^{\prime}\right|\right)$ for a given volume $d$. In the boundary layer, $v$ has to be chosen to be the minimizer of the elastic energy. If $h=\sum h_{i} \chi_{\left[a_{i}, b_{i}\right]}$, then set $\ell_{i}:=b_{i}-a_{i}$. The minimal energy is given by

$$
\min _{\sum_{i} h_{i} \ell_{i}=1} \frac{C_{W}}{2} \sum_{i} \ell_{i}^{2}+\sum_{i} h_{i}
$$


Assume, for the sake of contradiction, that two of the $\ell_{i}$ are non-zero, say $\ell_{1} \geq \ell_{2}>0$. For $\eta \in\left[-h_{1}, \frac{\ell_{2}}{\ell_{1}} h_{2}\right]$ consider $h_{1}+\eta$ and $h_{2}-\eta \frac{\ell_{1}}{\ell_{2}}$. Since $\left(\ell_{i}, h_{i}\right)$ is minimizing and $\left(h_{1}+\eta\right) \ell_{1}+$ $\left(h_{2}-\eta \frac{\ell_{1}}{\ell_{2}}\right) \ell_{2}=h_{1} \ell_{1}+h_{2} \ell_{2}$, we find that

$$
\eta-\eta \frac{\ell_{1}}{\ell_{2}} \geq 0 \quad \text { for all } \eta \in\left[-h_{1}, \frac{\ell_{2}}{\ell_{1}} h_{2}\right],
$$

and hence $\ell_{1}=\ell_{2}$, from which we deduce that $\ell_{i}=\ell$ for every $i$. The minimization problem then reduces to

$$
\min _{\ell \leq 1} \frac{C_{W}}{2} N \ell^{2}+\frac{1}{\ell}
$$

where $N$ is the number of intervals where $h \neq 0$. The minimum is attained for $N=1$ and $\ell_{\min }=\min \left\{1,\left(\frac{1}{C_{W}}\right)^{1 / 3}\right\}$.

Remark 4.6. If $W^{\infty}(A)=|A|^{2}$, then

$$
C_{W} \leq \min _{u(x, 0)=(x, 0)} \int_{0}^{1} \int_{0}^{+\infty}|\nabla u|^{2} d y d x \leq \int_{0}^{1} \int_{0}^{1}(1-y)^{2}+x^{2} d x d y=\frac{2}{3}<1,
$$

where where the second inequality follows by choosing $u(x, y):=\left(x(1-y) \chi_{y \leq 1}, 0\right)$. In particular, $\ell_{\min }=1$, and the flat configuration is optimal.

\subsection{The elastic dominant regime $F_{d, e_{0}} \simeq e_{0}^{p / 3} d^{2 / 3}$}

We now turn to the case in which $e_{0} \rightarrow+\infty, \frac{e_{0}^{p}}{d} \rightarrow+\infty$ and $e_{0}^{p} d^{2} \rightarrow+\infty$. Note that this allows also for $d \rightarrow 0$. In this regime, the main contribution to the energy comes from the elastic part. Define $\eta:=\left(\frac{d}{e_{0}^{p}}\right)^{1 / 3} \rightarrow 0$, so that $F_{d, e_{0}} \simeq \frac{d}{\eta}$. We thus rescale the energy $F_{d, e_{0}}(u, h)$ by $\frac{d}{\eta}$, and consider

$$
F_{\eta}(u, h):=\eta\left[\int_{\Omega_{h}} W\left(\frac{\partial u}{\partial x}, \frac{1}{d} \frac{\partial u}{\partial y}\right) d x d y+\int_{0}^{1} \sqrt{\frac{1}{d^{2}}+\left|h^{\prime}\right|^{2}} d x\right] .
$$

We first give a compactness and a liminf inequality result.

Proposition 4.7. Let $\left\{\left(u_{\eta}, h_{\eta}\right)\right\}$ be such that $\sup F_{\eta}\left(u_{\eta}, h_{\eta}\right) \leq C$, and set $\mu_{\eta}:=h_{\eta} d x$. Then there exists a subsequence (not relabeled) such that $\left\{\mu_{\eta}\right\}$ weakly-* converges to $\mu:=\sum_{i=1}^{+\infty} d_{i} \delta_{c_{i}}$ where $d_{i}>0$ satisfy $\sum_{i=1}^{+\infty} d_{i}=1$. Moreover, there holds

$$
\liminf _{\eta \rightarrow 0} F_{\eta}\left(u_{\eta}, h_{\eta}\right) \geq 3 C_{W}^{1 / 3} \sum_{i=1}^{+\infty} d_{i}^{2 / 3} .
$$


Proof. Since $\mu_{\eta}$ is a probability measure for every $\eta$, there exists a subsequence (not relabeled) and a probability measure $\mu$ such that $\mu_{\eta}$ weakly-* converges to $\mu$. We use the notation from the proof of Theorem 3.1 (see Figure $3(\mathrm{~b})$ ), i.e., $y_{0}:=\frac{1}{\sqrt{e_{0}^{p / 3} d^{2 / 3}}}$, and $\ell:=$ $\mathcal{H}^{1}\left(\Omega_{h_{\eta}} \cap\left(I \times\left\{y_{0}\right\}\right)\right)$. Let $I_{\ell}^{\eta}:=\Omega_{h_{\eta}} \cap\left(I \times\left\{y_{0}\right\}\right), I_{\ell}^{\eta}=\bigcup_{i=1}^{+\infty}\left[a_{i}^{\eta}, b_{i}^{\eta}\right]$, be the decomposition into connected components, $\ell_{i}^{\eta}:=b_{i}^{\eta}-a_{i}^{\eta}, d_{i}^{\eta}:=\left|\Omega_{h_{\eta}} \cap\left(\left[a_{i}^{\eta}, b_{i}^{\eta}\right] \times\left[y_{0},+\infty\right)\right)\right|$, and assume that the $d_{i}^{\eta}$ are in descending order. The latter can be assumed since $d_{i} \rightarrow 0$ as $i \rightarrow+\infty$. Note that $1-\sum_{i} d_{i}^{\eta} \leq y_{0}$, and thus $\lim _{\eta \rightarrow 0} \sum_{i} d_{i}^{\eta}=1$. Let finally $d_{i}:=\lim _{\eta \rightarrow 0} d_{i}^{\eta}$ (which we can assume exists for every $i \in \mathbb{N}$ up to further extraction of a subsequence). Since $F_{d, e_{0}} \simeq e_{0}^{p / 3} d^{2 / 3}$, by Theorem 3.1, we deduce that $\max _{i} \ell_{i}^{\eta} \leq C \eta$ and thus $\left(b_{i}^{\eta}-a_{i}^{\eta}\right) \rightarrow 0$ for all $i$. Indeed, otherwise, for every $n>0$ there would exist $\eta_{n} \rightarrow 0$ such that $\ell_{1} \geq n \eta_{n}$, and hence inf $F_{d, e_{0}} \geq C n e_{0}^{p / 3} d^{2 / 3}$ which contradicts the upper bound. Therefore, we may assume that for some $c_{i} \in[0,1], a_{i}^{\eta} \rightarrow c_{i}$ and $b_{i}^{\eta} \rightarrow c_{i}$. Let $\Omega_{i}^{\eta}:=\Omega_{h_{\eta}} \cap\left(\left[a_{i}^{\eta}, b_{i}^{\eta}\right] \times[0,+\infty)\right)$ and

$$
F_{\eta}\left(u_{\eta}, h_{\eta}, \Omega_{i}^{\eta}\right):=\eta \int_{a_{i}^{\eta}}^{b_{i}^{\eta}} \sqrt{\frac{1}{d^{2}}+\left|h_{\eta}^{\prime}\right|^{2}} d x+\eta \int_{\Omega_{i}^{\eta}} W\left(\frac{\partial u_{\eta}}{\partial x}, \frac{1}{d} \frac{\partial u_{\eta}}{\partial y}\right) d x d y
$$

Then for every $i$ (see (2))

$$
\begin{aligned}
F_{\eta}\left(u_{\eta}, h_{\eta}, \Omega_{i}^{\eta}\right) & \geq \eta \int_{a_{i}^{\eta}}^{b_{i}^{\eta}}\left|h_{\eta}^{\prime}\right| d x+\eta \int_{\left[a_{i}^{\eta}, b_{i}^{\eta}\right] \times\left[0, y_{0}\right]} W\left(\frac{\partial u_{\eta}}{\partial x}, \frac{1}{d} \frac{\partial u_{\eta}}{\partial y}\right) d x d y \\
& \geq 2 \eta \frac{d_{i}^{\eta}}{\ell_{i}^{\eta}}+\frac{\eta}{d} \min _{u(x, 0)=e_{0}(x, 0)} \int_{\left[a_{i}^{\eta}, b_{i}^{\eta}\right] \times\left[0, d y_{0}\right]} W(\nabla u) d x d y \\
& \geq 2 \eta \frac{d_{i}^{\eta}}{\ell_{i}^{\eta}}+\frac{\eta}{d} e_{0}^{p}\left(\ell_{i}^{\eta}\right)^{2} \min _{v(x, 0)=(x, 0)} \int_{[0,1] \times\left[0, \frac{d y_{0}}{\ell_{i}^{\eta}}\right]} \frac{W\left(e_{0} \nabla v\right)}{e_{0}^{p}} d x d y \\
& \geq 2 \eta \frac{d_{i}^{\eta}}{\ell_{i}^{\eta}}+\frac{\eta}{d} e_{0}^{p}\left(\ell_{i}^{\eta}\right)^{2} \min _{v(x, 0)=(x, 0)} \int_{[0,1] \times\left[0, \sqrt{e_{0}^{p / 3} d^{2 / 3}}\right]} \frac{W\left(e_{0} \nabla v\right)}{e_{0}^{p}} d x d y \\
& =2 \eta \frac{d_{i}^{\eta}}{\ell_{i}^{\eta}}+\frac{\eta}{d} e_{0}^{p}\left(\ell_{i}^{\eta}\right)^{2} C_{W}(1-\psi(\eta)) \\
& \geq 3 C_{W}^{1 / 3}(1-\psi(\eta))^{1 / 3}\left(d_{i}^{\eta}\right)^{2 / 3},
\end{aligned}
$$

where $\psi(\eta) \rightarrow 0$ as $\eta \rightarrow 0$. Summing over $i$ and letting $\eta \rightarrow 0$, we obtain the liminf inequality.

Now, for every $\varepsilon>0$, let $V^{\varepsilon}:=\left\{i \in \mathbb{N}: d_{i}^{\eta}<\varepsilon\right\}$. Then

$$
\begin{aligned}
\sum_{i \in V^{\varepsilon}} d_{i}^{\eta}=\sum_{i \in V^{\varepsilon}}\left(d_{i}^{\eta}\right)^{1 / 3}\left(d_{i}^{\eta}\right)^{2 / 3} & \leq \varepsilon^{1 / 3} \sum_{i \in V^{\varepsilon}}\left(d_{i}^{\eta}\right)^{2 / 3} \\
& \leq C \varepsilon^{1 / 3} \sum_{i \in V^{\varepsilon}} F_{\eta}\left(u_{\eta}, h_{\eta}, \Omega_{i}^{\eta}\right) \\
& \leq C \varepsilon^{1 / 3} F_{\eta}\left(u_{\eta}, h_{\eta}\right) \leq C \varepsilon^{1 / 3}
\end{aligned}
$$


The number of islands with $d_{i}^{\eta}>\varepsilon$ is uniformly bounded by some constant $N_{\varepsilon} \leq \frac{1}{\varepsilon}$. For fixed $\varepsilon>0$ let $I^{\varepsilon}:=\left(\bigcup_{i \in V^{\varepsilon}}\left[a_{i}^{\eta}, b_{i}^{\eta}\right]\right)^{c}$ and $\mu_{\eta}^{\varepsilon}:=h_{\eta} \chi_{I^{\varepsilon}} d x$. Then $\left\{\mu_{\eta}^{\varepsilon}\right\}$ converges weakly-* to $\mu^{\varepsilon}:=\sum_{i=1}^{N_{\varepsilon}} d_{i} \delta_{c_{i}}$. Finally $\mu_{\varepsilon} \rightarrow \mu$ since for every $\phi \in C(I)$

$$
\left|\left(\mu^{\varepsilon}-\mu\right)(\phi)\right|=\lim _{\eta \rightarrow 0} \int_{I^{\varepsilon}} h_{\eta} \phi d x \leq C\|\phi\|_{\infty} \varepsilon^{1 / 3} .
$$

Since $\mu_{\varepsilon}$ weakly-* converges $\sum_{i \in \mathbb{N}} d_{i} \delta_{c_{i}}$, this ends the proof.

Remark 4.8. In contrast to [14], the structure of the limiting measure seems not to follow directly from the Second Concentration-Compactness Lemma by P.L. Lions (see [39]), but rather from the scaling of the energy.

We finally prove the corresponding limsup inequality.

Proposition 4.9. If $\mu:=\sum_{i=1}^{+\infty} d_{i} \delta_{c_{i}}$ then there exist a sequence $\left\{\left(u_{\eta}, h_{\eta}\right)\right\}$ of functions $u_{\eta} \in W^{1, p}\left(\Omega_{h_{\eta}} ; \mathbb{R}^{2}\right)$, and nonnegative Lipschitz functions $h_{\eta}$ such that $\mu_{\eta}:=h_{\eta} d x$ are probability measures that weakly-* converge to $\mu$, and

$$
\limsup _{\eta \rightarrow 0} F_{\eta}\left(u_{\eta}, h_{\eta}\right) \leq 3 C_{W}^{1 / 3} \sum_{i=1}^{+\infty} d_{i}^{2 / 3} .
$$

Proof. Every measure $\mu=\sum_{i=1}^{+\infty} d_{i} \delta_{c_{i}}$ can be approximated in energy by the measures $\mu_{N}:=$ $\sum_{i=1}^{N} d_{i} \delta_{c_{i}}$, and by slightly moving the points $c_{i}$, we may assume that $c_{i} \notin\{0,1\}$. For such measures, we construct a recovery sequence as follows: Set $\ell_{i}:=\left(\frac{d_{i}}{C_{W}}\right)^{1 / 3} \eta, h_{i}:=\frac{d_{i}}{\ell_{i}}$, and let $h_{\eta}$ a Lipschitz function close to $\sum_{i=1}^{N} h_{i} \chi_{\left(c_{i}-\ell_{i} / 2, c_{i}+\ell_{i} / 2\right)}$. Finally, choose $u_{\eta}$ to be the minimizer of the elastic energy in $\Omega_{h_{\eta}}$.

Note that the minimizer of the limit functional,

$$
\min \left\{\sum_{i=1}^{+\infty} d_{i}^{2 / 3}: \sum_{i=1}^{+\infty} d_{i}=1\right\},
$$

is given by a single Dirac mass, i.e., $d_{1}=1$ and $d_{i}=0$ for $i>1$.

Remark 4.10. (i) The limiting energy charges only Dirac masses. This can be interpreted as a confirmation of the formation of islands in case that the amplitude of the mismatch is large compared to the thickness of the film.

(ii) The limit functional is not defined on a function space, but on a space of measures. The idea to consider limits in such a way has been used, e.g., in the study of the OhtaKawasaki model (see $[14,30]$ ), in the study of vortices in Ginzburg-Landau model (see $[33,41]$ ), and is also behind the idea of the blow-up method of Fonseca and Müller (see $[23])$. 


\section{Scaling law in dimension three for the Dirichlet energy}

In this section, we show that in higher dimension, a scaling law similar to the one of Theorem 3.1 holds. Let $D=D(0,1) \subset \mathbb{R}^{2}$ be the unit disk (but it could be replaced by any smooth bounded domain) and consider

$$
F_{d, e_{0}}(u, h):=\int_{\Omega_{h}}|\nabla u|^{2} d x d y+\int_{D} \sqrt{1+|\nabla h|^{2}} d x
$$

where $\Omega_{h}:=\{(x, y): x \in D, 0 \leq y \leq h(x)\}, u(x, 0)=e_{0}(x, 0)$ for all $x \in D$, and $\int_{D} h d x=d$.

Proposition 5.1. Let $d \geq 2 \pi$. There exist constants $C_{1}, C_{2}>0$ such that

$$
C_{1} \max \left\{1, d, e_{0}^{1 / 2} d^{3 / 4}\right\} \leq \inf F_{d, e_{0}} \leq C_{2} \max \left\{1, d, e_{0}^{1 / 2} d^{3 / 4}\right\}
$$

Proof. The upper bound is very similar to the two dimensional case, replacing rectangles by cylinders.

For the lower bound, we proceed along the lines of the proof of Theorem 3.1. We assume that $e_{0}^{1 / 2} d^{3 / 4} \geq C \max \{1, d\}$. Let $\Omega:=\{x \in D: h(x)>1\}$. Then $\left|\Omega_{h} \cap\{y \geq 1\}\right| \geq d-\pi$ and thus

$$
F(u, h) \geq \int_{\Omega \times[0,1]}|\nabla u|^{2} d x d y+\int_{\Omega}|\nabla h| d x \geq C\left(\int_{\Omega \times[0,1]}|\nabla u|^{2} d x d y+d \lambda_{1}(\Omega)\right),
$$

where $\lambda_{1}(\Omega)$ is the Cheeger constant of $\Omega$ defined as (see for instance [3])

$$
\begin{aligned}
\lambda_{1}(\Omega) & :=\min _{\int_{\Omega} h d x=1} \int_{\Omega}|\nabla h| d x+\int_{\partial \Omega}|h| d x=\min _{E \subset \Omega} \frac{P(E)}{|E|} \\
& =\frac{1}{d} \min \left\{\int_{\Omega}|\nabla h|: \int_{\Omega} h=d \text { and } h=0 \text { on } \partial \Omega\right\},
\end{aligned}
$$

where $P(E)$ denotes the perimeter of the set $E$. For a fixed domain $\Omega \subset D$, we want to estimate from below

$$
\min _{u(x, 0)=(x, 0)} \int_{\Omega \times[0,1]}|\nabla u|^{2} d x d y .
$$

Let us notice that by reducing $\Omega$ we can assume that $\lambda_{1}(\Omega)=\frac{P(\Omega)}{|\Omega|}$ i.e., that $\Omega$ is calibrable (see for example [3]) since it would only lower the energy. Notice that $\Omega$ is then a connected set. We then consider the minimizer $u$ of this problem which satisfies,

$$
\begin{cases}-\Delta u=0 & \text { in } \Omega \times(0,1) \\ u(x, 0)=(x, 0) & \text { in } \Omega \\ \frac{\partial u}{\partial \nu}=0 & \text { in } \partial \Omega \times(0,1) \cup \Omega \times\{1\} .\end{cases}
$$


Let $\mu_{k}^{2}$ be the increasing eigenvalues of the Laplacian in $\Omega$ with Neumann boundary conditions (so that $\mu_{1}=0$ ) and let $\phi_{k}$ be the associated eigenfunctions (normalized so that $\int_{\Omega} \phi_{k}^{2}=1$ ). We can then decompose $u$ with respect to the basis $\phi_{k}$, i.e.,

$$
u(x, y)=\sum_{k \geq 1} u_{k}(y) \phi_{k}(x)
$$

where $u_{k}$ is a vector valued function. From the equation we get

$$
u_{k}^{\prime \prime}-\mu_{k}^{2} u_{k}=0 \quad u_{k}(0)=u_{k} \quad u_{k}^{\prime}(1)=0
$$

where $u_{k}=\int_{\Omega} u(x, 0) \phi_{k} d x$ and thus $u_{k}(y)=\alpha \exp \left(\mu_{k} y\right)+\beta \exp \left(-\mu_{k} y\right)$ so that we find

$$
u_{k}(y)=\frac{\exp \left(-2 \mu_{k}\right)}{1+\exp \left(-2 \mu_{k}\right)} u_{k} \exp \left(\mu_{k} y\right)+\frac{1}{1+\exp \left(-2 \mu_{k}\right)} u_{k} \exp \left(-\mu_{k} y\right)
$$

We then get that (using that $\int_{\Omega}\left|\nabla \phi_{k}\right|^{2} d x=\mu_{k}^{2}$ )

$$
\int_{\Omega \times[0,1]}|\nabla u|^{2} d x d y=\sum_{k \geq 1} \int_{0}^{1}\left|u_{k}^{\prime}(y)\right|^{2}+\mu_{k}^{2}\left|u_{k}(y)\right|^{2} d y=\sum_{k \geq 2} \frac{1-\exp \left(-2 \mu_{k}\right)}{1+\exp \left(-2 \mu_{k}\right)} \mu_{k}\left|u_{k}\right|^{2} .
$$

By Cheeger's inequality (see [38]), for $k \geq 2$

$$
\mu_{k} \geq \mu_{2} \geq \frac{1}{4} \lambda_{1}(\Omega)
$$

and since $\Omega \subset D$, we also have $\lambda_{1}(\Omega) \geq \lambda_{1}(D)$ and thus

$$
\int_{\Omega \times[0,1]}|\nabla u|^{2} d x d y \geq \frac{1-\exp \left(-\frac{\lambda_{1}(D)}{2}\right)}{1+\exp \left(-\frac{\lambda_{1}(D)}{2}\right)} \frac{\lambda_{1}(\Omega)}{4} \sum_{k \geq 2}\left|u_{k}\right|^{2} .
$$

By Plancherel formula, there holds

$$
\sum_{k \geq 2}\left|u_{k}\right|^{2}=\int_{\Omega}|x|^{2} d x-\frac{1}{|\Omega|} \sum_{i=1}^{n}\left(\int_{\Omega} x_{i} d x\right)^{2} .
$$

Since

$$
\int_{\Omega}|x|^{2} d x-\frac{1}{|\Omega|} \sum_{i=1}^{n}\left(\int_{\Omega} x_{i} d x\right)^{2}=\frac{1}{2|\Omega|} \int_{\Omega \times \Omega}|x-z|^{2} d x d z,
$$

equation (14) transforms into

$$
\int_{\Omega \times[0,1]}|\nabla u|^{2} d x d y \geq C \lambda_{1}(\Omega) \frac{1}{|\Omega|} \int_{\Omega \times \Omega}|x-z|^{2} d x d z .
$$

It thus suffices to show the lower bound

$$
e_{0}^{2} \lambda_{1}(\Omega) \frac{1}{|\Omega|} \int_{\Omega \times \Omega}|x-z|^{2} d x d z+d \lambda_{1}(\Omega) \geq C e_{0}^{1 / 2} d^{3 / 4} .
$$


For that, we employ the interpolation inequality (recall that $\lambda_{1}(\Omega)=\frac{P(\Omega)}{|\Omega|}$ )

$$
P(\Omega)^{4 / 5}\left(\int_{\Omega \times \Omega}|x-z|^{2} d x d z\right)^{1 / 5} \geq C|\Omega|,
$$

which corresponds to $[34,(5.3)]$ with $\alpha=-2$, since their proof carries over verbatim to this choice. Finally, dividing (15) by $|\Omega|$, raising it to the power $5 / 4$, and multiplying it by $e_{0}^{1 / 2} d^{3 / 4}$, yields the desired lower bound.

Remark 5.2. (i) Notice that

$$
\min _{u(x, 0)=(x, 0)} \int_{\Omega \times[0,1]}|\nabla u|^{2} d x d y \simeq|(x, 0)|_{H^{1 / 2}(\Omega)}^{2}=\sum_{i} \int_{\Omega_{i} \times \Omega_{i}} \frac{1}{|x-z|} d x d z,
$$

where the sets $\Omega_{i}$ are the connected components of $\Omega$. Hence (at least formally), the right handside of (13) looks very similar to the Ohta-Kawasaki functional which has received a lot of attention in the last few years (see [34, 16, 14, 30, 2]).

(ii) The proof of Theorem 5.1 can be extended similarly to any space dimension.

(iii) In order to remove the condition $d \geq 2 \pi$, we would need the reverse of Cheeger's inequality. Unfortunately, it is known that Buser's inequality does not hold for problems with Neumann boundary conditions (see [38]).

\section{Acknowledgments}

Both authors thank I. Fonseca and G. Leoni for bringing this problem to their attention, for helpful discussions and for their constant support. They thank G. De Philippis and H. Knüpfer for interesting discussions concerning the last part of the paper, and warmly thank the Center for Nonlinear Analysis (NSF Grant No. DMS-0635983), where this research was carried out. The second author was partly funded by a postdoctoral fellowship of the National Science Foundation under Grant No. DMS-0905778. The final stages of this work were carried out while the first author was funded by a Von Humboldt postdoctoral fellowship.

\section{References}

[1] R. A. Adams and J. J. F. Fournier. Sobolev spaces, volume 140 of Pure and Applied Mathematics (Amsterdam). Elsevier/Academic Press, Amsterdam, second edition, 2003.

[2] G. Alberti, R. Choksi, and F. Otto. Uniform energy distribution for an isoperimetric problem with long-range interactions. J. Amer. Math. Soc., 22(2):569-605, 2009.

[3] F. Alter and V. Caselles. Uniqueness of the Cheeger set of a convex body. Nonlinear Anal., 70(1):32-44, 2009. 
[4] L. Ambrosio, N. Fusco, and D. Pallara. Functions of bounded variation and free discontinuity problems. Oxford Mathematical Monographs. The Clarendon Press Oxford University Press, New York, 2000.

[5] G. Anzellotti, S. Baldo, and D. Percivale. Dimension reduction in variational problems, asymptotic development in $\Gamma$-convergence and thin structures in elasticity. Asymptotic Anal., 9(1):61-100, 1994.

[6] P. Bella, M. Goldman, and B. Zwicknagl. Study of island formation in epitaxially strained films on unbounded domains. In preparation, 2013.

[7] P. Bella and R.V. Kohn. Wrinkles as a relaxation of compressive stresses in an annular thin film. To be published in Comm. Pure Appl. Math., 2013.

[8] H. Ben Belgacem. Relaxation of singular functionals defined on Sobolev spaces. ESAIM Control Optim. Calc. Var., 5:71-85 (electronic), 2000.

[9] E. Bonnetier and A. Chambolle. Computing the equilibrium configuration of epitaxially strained crystalline films. SIAM J. Appl. Math., 62(4):1093-1121 (electronic), 2002.

[10] A. Braides. Г-convergence for beginners, volume 22 of Oxford Lecture Series in Mathematics and its Applications. Oxford University Press, Oxford, 2002.

[11] A. Braides, A. Chambolle, and M. Solci. A relaxation result for energies defined on pairs set-function and applications. ESAIM Control Optim. Calc. Var., 13(4):717-734 (electronic), 2007.

[12] A. Chambolle and C. J. Larsen. $C^{\infty}$ regularity of the free boundary for a two-dimensional optimal compliance problem. Calc. Var. Partial Differential Equations, 18(1):77-94, 2003.

[13] A. Chambolle and M. Solci. Interaction of a bulk and a surface energy with a geometrical constraint. SIAM J. Math. Anal., 39:77-102, 2007.

[14] R. Choksi and M. A. Peletier. Small volume fraction limit of the diblock copolymer problem: I. Sharp-interface functional. SIAM J. Math. Anal., 42(3):1334-1370, 2010.

[15] P. G. Ciarlet. Mathematical elasticity. Vol. II, volume 27 of Studies in Mathematics and its Applications. North-Holland Publishing Co., Amsterdam, 1997. Theory of plates.

[16] Marco Cicalese and Emanuele Spadaro. Droplet minimizers of an isoperimetric problem with long-range interactions. Comm. Pure Appl. Math., 66(8):1298-1333, 2013.

[17] S. Conti. A lower bound for a variational model for pattern formation in shape-memory alloys. Contin. Mech. Thermodyn., 17(6):469-476, 2006.

[18] G. Dal Maso. An introduction to $\Gamma$-convergence. Progress in Nonlinear Differential Equations and their Applications, 8. Birkhäuser Boston Inc., Boston, MA, 1993. 
[19] G. Dal Maso, I. Fonseca, and G. Leoni. Nonlocal character of the reduced theory of thin films with higher order perturbations. Adv. Calc. Var., 3(3):287-319, 2010.

[20] I. Fonseca, N. Fusco, G. Leoni, and M. Morini. Equilibrium configurations of epitaxially strained crystalling films: Existence and regularity results. Arch. Rat. Mech. Anal., 186:477-537, 2007.

[21] I. Fonseca, N. Fusco, G. Leoni, and M. Morini. Motion of elastic thin films by anisotropic surface diffusion with curvature regularization. Archive for Rational Mechanics and Analysis, pages 1-42, 2012.

[22] I. Fonseca and S. Müller. Quasiconvex integrands and lower semicontinuity in $\mathrm{L}^{1}$. SIAM J. Math. Anal., 23:1081-1098, 1992.

[23] I. Fonseca and S. Müller. Relaxation of quasiconvex functionals in $\operatorname{BV}\left(\Omega, \mathbf{R}^{p}\right)$ for integrands $f(x, u, \nabla u)$. Arch. Rational Mech. Anal., 123(1):1-49, 1993.

[24] G. Friesecke, R. D. James, and S. Müller. A theorem on geometric rigidity and the derivation of nonlinear plate theory from three-dimensional elasticity. Comm. Pure Appl. Math., 55(11):1461-1506, 2002.

[25] G. Friesecke, R. D. James, and S. Müller. A hierarchy of plate models derived from nonlinear elasticity by gamma-convergence. Arch. Ration. Mech. Anal., 180(2):183-236, 2006 .

[26] N. Fusco and M. Morini. Equilibrium configurations of epitaxially strained elastic films: Second order minimality conditions and qualitative properties of solutions. Arch. Rat. Mech. Anal., 203:247-327, 2012.

[27] H. Gao and W.D. Nix. Surface roughening of heteroepitaxial thin films. Annual Review of Materials Science, 29:173-209, 1999.

[28] M. Giaquinta, G. Modica, and J. Souček. Functionals with linear growth in the calculus of variations. I, II. Comment. Math. Univ. Carolin., 20(1):143-156, 157-172, 1979.

[29] E. Giusti. Direct methods in the calculus of variations. World Scientific Publishing Co. Inc., River Edge, NJ, 2003.

[30] Dorian Goldman, Cyrill B. Muratov, and Sylvia Serfaty. The $\Gamma$-Limit of the TwoDimensional Ohta-Kawasaki Energy. I. Droplet Density. Arch. Ration. Mech. Anal., 210(2):581-613, 2013.

[31] J.L. Gray, R. Hull, and J.A. Floro. Formation of one-dimensional surface grooves from pit instabilities in annealed SiG/ Si (100) epitaxial films. Applied physics letters, 85(15), 2004 . 
[32] P. Hornung. A $\Gamma$-convergence result for thin martensitic films in linearized elasticity. SIAM J. Math. Anal., 40(1):186-214, 2008.

[33] R. L. Jerrard and H. M. Soner. The Jacobian and the Ginzburg-Landau energy. Calc. Var. Partial Differential Equations, 14(2):151-191, 2002.

[34] H. Knüpfer and C. Muratov. On an isoperimetric problem with a competing nonlocal term I: The planar case. Comm. Pure Appl. Math., 66(7):1129-1162, 2013.

[35] R. V. Kohn and S. Müller. Surface energy and microstructure in coherent phase transitions. Comm. Pure Appl. Math., 47(4):405-435, 1994.

[36] V. A. Kondrat'ev and O. A. Oleŭnik. Boundary value problems for a system in elasticity theory in unbounded domains. Korn inequalities. Uspekhi Mat. Nauk, 43(5(263)):55-98, $239,1988$.

[37] H. Le Dret and A. Raoult. The nonlinear membrane model as variational limit of nonlinear three-dimensional elasticity. J. Math. Pures Appl. (9), 74(6):549-578, 1995.

[38] M. Ledoux. A simple analytic proof of an inequality by P. Buser. Proc. Amer. Math. Soc., 121(3):951-959, 1994.

[39] P.-L. Lions. The concentration-compactness principle in the calculus of variations. The limit case. I. Rev. Mat. Iberoamericana, 1(1):145-201, 1985.

[40] P. Piovano. Evolution of elastic thin films with curvature regularization via minimizing movements. published online in Cal. Var. Partial Differential Equations, 2013.

[41] E. Sandier and S. Serfaty. Vortices in the magnetic Ginzburg-Landau model. Progress in Nonlinear Differential Equations and their Applications, 70. Birkhäuser Boston Inc., Boston, MA, 2007.

[42] B. J. Spencer. Asymptotic derivation of the glued-wetting-layer model and contact-angle condition for stranski-krastanow islands. Phys. Rev. B, 59:2011-2017, Jan 1999.

[43] B. J. Spencer and J. Tersoff. Asymmetry and shape transitions of epitaxially strained islands on vicinal surfaces. Appl. Phys. Lett., 96:073114, 2010. 